TRANSACTIONS OF THE

AMERICAN MATHEMATICAL SOCIETY

Volume 365, Number 12, December 2013, Pages 6283-6307

S 0002-9947(2013)05886-2

Article electronically published on June 3, 2013

\title{
NEUMANN FUNCTIONS FOR SECOND ORDER ELLIPTIC SYSTEMS WITH MEASURABLE COEFFICIENTS
}

\author{
JONGKEUN CHOI AND SEICK KIM
}

\begin{abstract}
We study Neumann functions for divergence form, second-order elliptic systems with bounded measurable coefficients in a bounded Lipschitz domain or a Lipschitz graph domain. We establish existence, uniqueness, and various estimates for the Neumann functions under the assumption that weak solutions of the system enjoy interior Hölder continuity. Also, we establish global pointwise bounds for the Neumann functions under the assumption that weak solutions of the system satisfy a certain natural local boundedness estimate. Moreover, we prove that such a local boundedness estimate for weak solutions of the system is in fact equivalent to the global pointwise bound for the Neumann function. We present a unified approach valid for both the scalar and the vectorial cases.
\end{abstract}

\section{INTRODUCTION}

In this article, we are concerned with Neumann functions (sometimes called Neumann Green's function) for divergence form, second-order elliptic systems with bounded measurable coefficients in a bounded Lipschitz domain or a Lipschitz graph domain. More precisely, we consider Neumann functions for the $m \times m$ elliptic systems

$$
\sum_{j=1}^{m} L_{i j} u^{j}:=-\sum_{j=1}^{m} \sum_{\alpha, \beta=1}^{d} D_{\alpha}\left(A_{i j}^{\alpha \beta}(x) D_{\beta} u^{j}\right), \quad i=1, \ldots, m
$$

in $\Omega$, where $\Omega$ is a bounded Lipschitz domain or a Lipschitz graph domain in $\mathbb{R}^{d}$ with $d \geq 3$. Here, we assume that the coefficients are measurable functions defined in the whole space $\mathbb{R}^{d}$ satisfying the strong ellipticity and the uniform boundedness condition; see Section 2 for their precise definitions. We do not assume that the coefficients of system (1.1) are symmetric. We will later impose some further assumptions on system (1.1) in the case when $m>1$ but not explicitly on its coefficients.

Analogous to the role of Green's functions in the study of the Dirichlet boundary value problem of elliptic equations, Neumann functions play a significant role in the study of the Neumann boundary value problem. For this reason, the Neumann functions are discussed in many papers, but, however, with only a few exceptions, it is assumed that the coefficients and the domains are sufficiently regular. In the case when $m=1$, Kenig and Pipher [16] constructed Neumann functions for the divergence form elliptic equations with $L^{\infty}$ coefficients and derived various estimates

Received by the editors July 2, 2011 and, in revised form, March 11, 2012.

2010 Mathematics Subject Classification. Primary 35J08, 35J47, 35J57.

Key words and phrases. Neumann function, Green's function, Neumann boundary problem, second-order elliptic system, measurable coefficients. 
for the Neumann functions in the unit ball $B$. Those estimates are the same sort of estimates known for the Green's functions that appear in [11, 17] and are nicely summarized in [14, Theorem 1.6.3]. Their methods of proof are general enough to allow $B$ to be a bounded star-like Lipschitz domain, but, however, as pointed out in [19], it is not immediately clear whether they also work for general bounded Lipschitz domains. Also, their methods do not seem to work for unbounded domains such as the half space. On the other hand, Hofmann and Kim 12 recently proved existence and various (interior) estimates for the Green's function of system (1.1) in arbitrary domains under the assumption that weak solutions of system (1.1) satisfy an interior Hölder continuity estimate. Their result has been complemented by a very recent article by Kang and Kim [13, where global estimates of Green's functions for system (1.1) are established under some other (but similar) assumptions. In the case when $m=1$, the De Giorgi-Moser-Nash theory for weak solutions implies such estimates, and thus, in particular, they were able to reproduce the related classical results of [11, 17.

The goal of this article is to present a unified approach for the construction and estimates of Neumann functions of the elliptic systems (1.1) in a bounded Lipschitz domain as well as in an unbounded domain above a Lipschitz graph. As a matter of fact, it is exactly where the strength of our paper lies. By using our unified method, we reproduce the estimates for Neumann functions of scalar equations with $L^{\infty}$ coefficients in the unit ball presented in [16], as well as those for systems with $C^{\alpha}$ coefficients in $C^{1, \alpha}$ domains appearing in a recent article [15]. Recently, there has been some interest in studying boundary value problems for divergence form elliptic equations with complex $L^{\infty}$ coefficients above a Lipschitz graph; see, e.g., [1, 2, 3. In this context, it is natural to consider Green's functions and Neumann functions for elliptic systems with $L^{\infty}$ coefficients in a Lipschitz graph domain. In fact, properties of Green's function investigated in [12, for elliptic equations whose coefficients are complex perturbations of real $L^{\infty}$ coefficients, were used in [1]. However, we are not even able to find literature dealing with Neumann functions in the half space for scalar elliptic equations with $L^{\infty}$ coefficients. As we have already mentioned, our method also goes through in that case, and, in particular, we derive the estimates for the Neumann function of the scalar elliptic equations with $L^{\infty}$ coefficients in a Lipschitz graph domain that corresponds to the estimates in 14, Theorem 1.6.3]. We hope this article may serve as a reference for the Neumann functions and their properties so that it may become a useful tool for other authors.

We shall now briefly describe our main result. Let $\Omega \subset \mathbb{R}^{d}$ with $d \geq 3$ be a bounded Lipschitz domain or a Lipschitz graph domain. We first construct the Neumann function of the system (1.1) under the assumption that its weak solutions are locally Hölder continuous. In doing so, we also derive various interior estimates for the Neumann function; see Theorems 3.1] and 5.1. We then show that if system (1.1) has such a property that weak solutions of Neumann problems with nice data are locally bounded and satisfy a certain natural estimate (see conditions $(\underline{\mathrm{LB}})$ and $\left(\underline{\mathrm{LB}^{\prime}}\right)$ in Sections 3 and 5 , then its Neumann function $\mathbf{N}(x, y)$ has the following global pointwise bound; see Theorems 3.6 and 5.2 .

$$
|\boldsymbol{N}(x, y)| \leq C|x-y|^{2-d}, \quad \forall x, y \in \Omega, \quad x \neq y .
$$

Conversely, if the Neumann function has the above pointwise bound, then we prove that the system should satisfy the aforementioned local boundedness property; see Theorems 3.9 and 5.2. An immediate consequence of our results combined with 
the celebrated De Giorgi-Moser-Nash theory would be that the Neumann function of scalar elliptic equations (i.e., $m=1$ ) enjoy the pointwise estimate (1.2) if $\Omega$ is a bounded Lipschitz domain or a Lipschitz graph domain. Moreover, if the coefficients of system (1.1) belong to the VMO class and $\Omega$ is a bounded $C^{1}$ domain, then $W^{1, p}$ estimates imply the aforementioned local boundedness property, and thus we would have the estimate (1.2) in that case too. As a matter of fact, in those cases we also have

$$
\begin{aligned}
& \left|\boldsymbol{N}(x, y)-\boldsymbol{N}\left(x^{\prime}, y\right)\right| \leq C\left|x-x^{\prime}\right|^{\mu}|x-y|^{2-d-\mu} \text { if } x \neq y \text { and } 2\left|x-x^{\prime}\right|<|x-y|, \\
& \left|\boldsymbol{N}(x, y)-\boldsymbol{N}\left(x, y^{\prime}\right)\right| \leq C\left|y-y^{\prime}\right|^{\mu}|x-y|^{2-d-\mu} \text { if } x \neq y \text { and } 2\left|y-y^{\prime}\right|<|x-y|
\end{aligned}
$$

for some $\mu \in(0,1]$; see Remark 3.8

The organization of the paper is as follows. In Section 2, we introduce some notation and definitions including weak formulations of Neumann problems and the precise definition of Neumann functions of system (1.1). In Section 3, we state our main theorems, including existence and global pointwise estimates for Neumann functions in bounded Lipschitz domains, and their proofs are presented in Section 4 , Section 5 is devoted to the study of the Neumann function in a Lipschitz graph domain. In the Appendix we provide the proofs of some technical lemmas.

Finally, a few remarks are in order. This article is, in spirit, very similar to [12, 13, where corresponding results for Green's functions have been established. However, the technical details are very different since the Neumann boundary condition is more difficult to handle than the Dirichlet condition. For instance, in [12, the Green's functions are constructed in arbitrary domains, but here Neumann functions are constructed only in domains with Lipschitz boundary. We do not treat the case $d=2$ in our paper. In dimension two, the Neumann functions should have logarithmic growth and require some other methods. As a matter of fact, our method breaks down and is not applicable in the two dimensional case. One way to overcome this difficulty is to utilize the so-called Neumann heat kernel of the elliptic operator defined in a Lipschitz cylinder $\Omega \times(0, \infty) \subset \mathbb{R}^{3}$. However, this approach requires first establishing a pointwise bound for the Neumann heat kernel that is sharp enough to be integrable in the $t$-variable; see [6, 7. for the treatment of Green's functions of elliptic systems in two dimensional domains. This topic will be discussed elsewhere because the Neumann heat kernel is an interesting subject in its own right. After our submission of the first version of this paper, Taylor et al. 20] constructed the Green's function for the mixed problem for elliptic systems in two dimensions.

\section{Preliminaries}

2.1. Basic notation. We mainly follow the notation used in 12 , 13 . Let $d \geq 3$ be an integer. We recall that a function $\varphi: \mathbb{R}^{d-1} \rightarrow \mathbb{R}$ is Lipschitz if there exists a constant $K<\infty$ such that

$$
\left|\varphi\left(x^{\prime}\right)-\varphi\left(y^{\prime}\right)\right| \leq K\left|x^{\prime}-y^{\prime}\right|, \quad \forall x^{\prime}, y^{\prime} \in \mathbb{R}^{d-1} .
$$

A bounded domain $\Omega \subset \mathbb{R}^{d}$ is called a Lipschitz domain if $\partial \Omega$ locally is given by the graph of a Lipschitz function. A domain $\Omega \subset \mathbb{R}^{d}$ is called a Lipschitz graph domain if

$$
\Omega=\left\{x=\left(x^{\prime}, x_{d}\right) \in \mathbb{R}^{d}: x_{d}>\varphi\left(x^{\prime}\right)\right\},
$$


where $\varphi: \mathbb{R}^{d-1} \rightarrow \mathbb{R}$ is a Lipschitz function. Throughout the entire article, we let $\Omega$ be a Lipschitz domain or Lipschitz graph domain in $\mathbb{R}^{d}$.

For $p \geq 1$ and $k$ a nonnegative integer, we denote by $W^{k, p}(\Omega)$ the usual Sobolev space. When $\Omega$ is a Lipschitz domain, we define the space $\tilde{W}^{1,2}(\Omega)$ as the family of all functions $u \in W^{1,2}(\Omega)$ satisfying $\int_{\partial \Omega} u=0$ in the sense of trace. We warn the reader that the space $\tilde{W}^{1,2}(\Omega)$ is different from the space $\tilde{W}_{1}^{2}(B)$ used in [16]. By using the Rellich-Kondrachov compactness theorem, one can easily show that there is a constant $C=C(d, \Omega)$ such that

$$
\|u\|_{L^{2}(\Omega)} \leq C\|D u\|_{L^{2}(\Omega)}, \quad \forall u \in \tilde{W}^{1,2}(\Omega) .
$$

The space $Y^{1,2}(\Omega)$ is defined as the family of all weakly differentiable functions $u \in L^{2 d /(d-2)}(\Omega)$ whose weak derivatives are functions in $L^{2}(\Omega)$. The space $Y^{1,2}(\Omega)$ is endowed with the norm

$$
\|u\|_{Y^{1,2}(\Omega)}:=\|u\|_{L^{2 d /(d-2)}(\Omega)}+\|D u\|_{L^{2}(\Omega)} .
$$

For a Lipschitz graph domain $\Omega$ with Lipschitz constant $K$, it is easy to show (see the Appendix) that the following Sobolev inequality holds:

$$
\|u\|_{L^{2 d /(d-2)}(\Omega)} \leq C(d, K)\|D u\|_{L^{2}(\Omega)}, \quad \forall u \in Y^{1,2}(\Omega) .
$$

We denote $\Omega_{R}(x)=\Omega \cap B_{R}(x)$ and $\Sigma_{R}(x)=\partial \Omega \cap B_{R}(x)$ for any $R>0$. We abbreviate $\Omega_{R}=\Omega_{R}(x)$ and $\Sigma_{R}=\Sigma_{R}(x)$ if the point $x$ is well understood in the context. We define $d_{x}=\operatorname{dist}(x, \partial \Omega)=\inf \{|x-y|: y \in \partial \Omega\}$.

2.2. Elliptic systems. Let $L$ be an elliptic operator acting on column vector valued functions $\boldsymbol{u}=\left(u^{1}, \ldots, u^{m}\right)^{T}$ defined on a subset of $\mathbb{R}^{d}$ in the following way:

$$
L \boldsymbol{u}=-D_{\alpha}\left(\boldsymbol{A}^{\alpha \beta} D_{\beta} \boldsymbol{u}\right),
$$

where we use the usual summation convention over repeated indices $\alpha, \beta=1, \ldots, d$, and $\boldsymbol{A}^{\alpha \beta}=\boldsymbol{A}^{\alpha \beta}(x)$ are $m \times m$ matrix valued functions defined on the whole space $\mathbb{R}^{d}$ with entries $A_{i j}^{\alpha \beta}$ that satisfy the strong ellipticity condition

$$
A_{i j}^{\alpha \beta}(x) \xi_{\beta}^{j} \xi_{\alpha}^{i} \geq \lambda|\boldsymbol{\xi}|^{2}:=\lambda \sum_{i=1}^{m} \sum_{\alpha=1}^{d}\left|\xi_{\alpha}^{i}\right|^{2}, \quad \forall \boldsymbol{\xi} \in \mathbb{R}^{m d}, \quad \forall x \in \mathbb{R}^{d},
$$

for some constant $\lambda>0$, and also the uniform boundedness condition

$$
\left|A_{i j}^{\alpha \beta}(x) \xi_{\alpha}^{j} \eta_{\beta}^{i}\right| \leq M|\boldsymbol{\xi}||\boldsymbol{\eta}|, \quad \forall \boldsymbol{\xi}, \boldsymbol{\eta} \in \mathbb{R}^{m d}, \quad \forall x \in \mathbb{R}^{d},
$$

for some constant $M>0$. Notice that the $i$-th component of the column vector $L \boldsymbol{u}$ coincides with $L_{i j} u^{j}$ in (1.1). The adjoint operator ${ }^{t} L$ is defined by

$$
{ }^{t} L \boldsymbol{u}=-D_{\alpha}\left({ }^{t} \boldsymbol{A}^{\alpha \beta} D_{\beta} \boldsymbol{u}\right),
$$

where ${ }^{t} \boldsymbol{A}^{\alpha \beta}=\left(\boldsymbol{A}^{\beta \alpha}\right)^{T}$; i.e., ${ }^{t} A_{i j}^{\alpha \beta}=A_{j i}^{\beta \alpha}$.

2.3. Neumann boundary value problem. We denote by $\boldsymbol{A} D \boldsymbol{u} \cdot \boldsymbol{n}$ the conormal derivative of $\boldsymbol{u}$ associated with the operator $L$; i.e., the $i$-th component of $\boldsymbol{A D} \boldsymbol{u} \cdot \boldsymbol{n}$ is defined by

$$
(\boldsymbol{A D} \boldsymbol{u} \cdot \boldsymbol{n})^{i}=A_{i j}^{\alpha \beta} D_{\beta} u^{j} n_{\alpha},
$$


where $\boldsymbol{n}=\left(n_{1}, \ldots, n_{d}\right)^{T}$ is the outward unit normal to $\partial \Omega$. Let $\Sigma$ be an open subset of $\partial \Omega$ and $\boldsymbol{f} \in L_{l o c}^{1}(\Omega)^{m}$ and $\boldsymbol{g} \in L_{l o c}^{1}(\Sigma)^{m}$. We shall say that $\boldsymbol{u} \in W_{l o c}^{1,1}(\Omega)^{m}$ is a weak solution of

$$
L \boldsymbol{u}=\boldsymbol{f} \text { in } \Omega, \quad \boldsymbol{A} D \boldsymbol{u} \cdot \boldsymbol{n}=\boldsymbol{g} \text { on } \Sigma
$$

if the following identity holds:

$$
\int_{\Omega} A_{i j}^{\alpha \beta} D_{\beta} u^{j} D_{\alpha} \phi^{i}-\int_{\Sigma} g^{i} \phi^{i}=\int_{\Omega} f^{i} \phi^{i}, \quad \forall \phi \in C_{c}^{\infty}(\Omega \cup \Sigma)^{m} .
$$

Observe that (2.5) makes sense if $\boldsymbol{f}$ is a vector valued measure in $\Omega$; see part ii) in the definition of a Neumann function below. We are mostly interested in the case when $\Sigma=\partial \Omega$.

2.3.1. Neumann problem in a bounded Lipschitz domain. Let $\Omega \subset \mathbb{R}^{d}$ be a bounded Lipschitz domain. Notice that inequality (2.1) implies that $\boldsymbol{H}:=\tilde{W}^{1,2}(\Omega)^{m}$ becomes a Hilbert space with the inner product

$$
\langle\boldsymbol{u}, \boldsymbol{v}\rangle_{\boldsymbol{H}}:=\int_{\Omega} D_{\alpha} u^{i} D_{\alpha} v^{i}
$$

If we define the bilinear form associated to the operator $L$ as

$$
B(\boldsymbol{u}, \boldsymbol{v}):=\int_{\Omega} A_{i j}^{\alpha \beta} D_{\beta} u^{j} D_{\alpha} v^{i},
$$

then by (2.3) and (2.4) the bilinear form $B$ becomes coercive and bounded on $\boldsymbol{H}$. Observe that by inequality (2.1) and the Sobolev imbedding theorem, we have

$$
\|\boldsymbol{u}\|_{L^{2 d /(d-2)}(\Omega)} \leq C\|D \boldsymbol{u}\|_{L^{2}(\Omega)}=C\|\boldsymbol{u}\|_{\boldsymbol{H}}, \quad \forall \boldsymbol{u} \in \boldsymbol{H}=\tilde{W}^{1,2}(\Omega)^{m} .
$$

Let $\boldsymbol{f} \in L^{2 d /(d+2)}(\Omega)^{m}$ and $\boldsymbol{g} \in L^{2}(\partial \Omega)^{m}$ satisfy the "compatibility" condition

$$
\int_{\Omega} \boldsymbol{f}+\int_{\partial \Omega} \boldsymbol{g}=0
$$

Then, by inequality (2.8) and the trace theorem combined with (2.1), we find that

$$
F(\boldsymbol{u}):=\int_{\Omega} \boldsymbol{f} \cdot \boldsymbol{u}+\int_{\partial \Omega} \boldsymbol{g} \cdot \boldsymbol{u}
$$

is a bounded linear functional on $\boldsymbol{H}$. Therefore, the Lax-Milgram theorem implies that there exists a unique $\boldsymbol{u}$ in $\boldsymbol{H}$ such that $B(\boldsymbol{u}, \boldsymbol{v})=F(\boldsymbol{v})$ for all $\boldsymbol{v} \in \boldsymbol{H}=$ $\tilde{W}^{1,2}(\Omega)^{m}$. Observe that any function $\boldsymbol{v} \in W^{1,2}(\Omega)^{m}$ is represented as a sum of a function in $\boldsymbol{H}$ and a constant vector in $\mathbb{R}^{m}$ as follows:

$$
\boldsymbol{v}=\left(\boldsymbol{v}-f_{\partial \Omega} \boldsymbol{v}\right)+f_{\partial \Omega} \boldsymbol{v}=: \tilde{\boldsymbol{v}}+\boldsymbol{c}
$$

Notice that condition (2.9) implies $F(\boldsymbol{c})=0$. Then the identity $B(\boldsymbol{u}, \tilde{\boldsymbol{v}})=F(\tilde{\boldsymbol{v}})$ yields

$$
\int_{\Omega} A_{i j}^{\alpha \beta} D_{\beta} u^{j} D_{\alpha} v^{i}=\int_{\Omega} f^{i} v^{i}+\int_{\partial \Omega} g^{i} v^{i}, \quad \forall \boldsymbol{v} \in W^{1,2}(\Omega)^{m} .
$$

Therefore, we have a unique solution $\boldsymbol{u}$ in $\boldsymbol{H}=\tilde{W}^{1,2}(\Omega)^{m}$ of the Neumann problem

$$
\left\{\begin{aligned}
L \boldsymbol{u}=\boldsymbol{f} & \text { in } \Omega, \\
\boldsymbol{A D \boldsymbol { u } \cdot \boldsymbol { n }}=\boldsymbol{g} & \text { on } \partial \Omega,
\end{aligned}\right.
$$

provided $\boldsymbol{f} \in L^{2 d /(d+2)}(\Omega)^{m}$ and $\boldsymbol{g} \in L^{2}(\partial \Omega)^{m}$ satisfy the compatibility condition (2.9). 
2.3.2. Neumann problem in a Lipschitz graph domain. Let $\Omega \subset \mathbb{R}^{d}$ be a Lipschitz graph domain and recall inequality (2.2). Similar to the bounded Lipschitz domain case, $\boldsymbol{H}:=Y^{1,2}(\Omega)^{m}$ becomes a Hilbert space with the inner product (2.6). Also, the bilinear form $B$ in (2.7) is coercive and bounded in $Y^{1,2}(\Omega)^{m}$. For any $\boldsymbol{f} \in$ $L^{2 d /(d+2)}(\Omega)^{m}$, inequality (2.2) implies that

$$
F(\boldsymbol{v}):=\int_{\Omega} f^{i} v^{i}
$$

is a bounded linear functional on $Y^{1,2}(\Omega)^{m}$. Therefore, the Lax-Milgram theorem implies that there exists a unique $\boldsymbol{u}$ in $Y^{1,2}(\Omega)^{m}$ such that $B(\boldsymbol{u}, \boldsymbol{v})=F(\boldsymbol{v})$ for all $\boldsymbol{v} \in Y^{1,2}(\Omega)^{m}$; i.e., we have

$$
\int_{\Omega} A_{i j}^{\alpha \beta} D_{\beta} u^{j} D_{\alpha} v^{i}=\int_{\Omega} f^{i} v^{i}, \quad \forall \boldsymbol{v} \in Y^{1,2}(\Omega)^{m} .
$$

Hereafter, we shall say that $\boldsymbol{u}$ is a unique solution in $Y^{1,2}(\Omega)^{m}$ of the Neumann problem

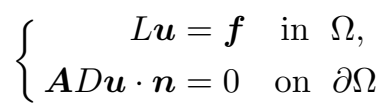

if $\boldsymbol{u} \in Y^{1,2}(\Omega)^{m}$ and satisfies identity (2.11).

2.4. Neumann function. In the definitions below, $\boldsymbol{N}=\boldsymbol{N}(x, y)$ will be an $m \times m$ matrix valued function with measurable entries $N_{i j}: \Omega \times \Omega \rightarrow \overline{\mathbb{R}}$.

2.4.1. Neumann function in a bounded Lipschitz domain. We say that $\boldsymbol{N}$ is a $\mathrm{Neu}-$ mann function of $L$ in a bounded Lipschitz domain $\Omega$ if it satisfies the following properties:

i) $\boldsymbol{N}(\cdot, y) \in W_{l o c}^{1,1}(\Omega)$ and $\boldsymbol{N}(\cdot, y) \in W^{1,2}\left(\Omega \backslash B_{r}(y)\right)$ for all $y \in \Omega$ and $r>0$. Moreover, $\int_{\partial \Omega} \boldsymbol{N}(\cdot, y)=0$ in the sense of trace.

ii) $L \boldsymbol{N}(\cdot, y)=\delta_{y} \boldsymbol{I}$ in $\Omega$ and $\boldsymbol{A} D \boldsymbol{N}(\cdot, y) \cdot \boldsymbol{n}=-\frac{1}{|\partial \Omega|} \boldsymbol{I}$ on $\partial \Omega$ for all $y \in \Omega$ in the sense that

$$
\int_{\Omega} A_{i j}^{\alpha \beta} D_{\beta} N_{j k}(\cdot, y) D_{\alpha} \phi^{i}+\frac{1}{|\partial \Omega|} \int_{\partial \Omega} \phi^{k}=\phi^{k}(y), \quad \forall \phi \in C^{\infty}(\bar{\Omega})^{m} .
$$

iii) For any $\boldsymbol{f}=\left(f^{1}, \ldots, f^{m}\right)^{T} \in C_{c}^{\infty}(\Omega)^{m}$, the function $\boldsymbol{u}$ given by

$$
\boldsymbol{u}(x):=\int_{\Omega} \boldsymbol{N}(y, x)^{T} \boldsymbol{f}(y) d y
$$

is a unique solution in $\tilde{W}^{1,2}(\Omega)^{m}$ of the problem

$$
\left\{\begin{aligned}
{ }^{t} L \boldsymbol{u} & =\boldsymbol{f} \quad \text { in } \Omega, \\
{ }^{t} \boldsymbol{A} D \boldsymbol{u} \cdot \boldsymbol{n} & =-\frac{1}{|\partial \Omega|} \int_{\Omega} \boldsymbol{f} \quad \text { on } \partial \Omega .
\end{aligned}\right.
$$

2.4.2. Neumann function in a Lipschitz graph domain. We say that $\boldsymbol{N}$ is a Neumann function of $L$ in a Lipschitz graph domain $\Omega$ if it satisfies the following properties:

i) $\boldsymbol{N}(\cdot, y) \in W_{l o c}^{1,1}(\Omega)$ and $\boldsymbol{N}(\cdot, y) \in Y^{1,2}\left(\Omega \backslash B_{r}(y)\right)$ for all $y \in \Omega$ and $r>0$. 
ii) $L \boldsymbol{N}(\cdot, y)=\delta_{y} \boldsymbol{I}$ in $\Omega$ and $\boldsymbol{A} D \boldsymbol{N}(\cdot, y) \cdot \boldsymbol{n}=0$ on $\partial \Omega$ for all $y \in \Omega$ in the sense that

$$
\int_{\Omega} A_{i j}^{\alpha \beta} D_{\beta} N_{j k}(\cdot, y) D_{\alpha} \phi^{i}=\phi^{k}(y), \quad \forall \phi=\left(\phi^{1}, \ldots, \phi^{m}\right)^{T} \in C_{c}^{\infty}(\bar{\Omega})^{m} .
$$

iii) For any $\boldsymbol{f} \in C_{c}^{\infty}(\Omega)^{m}$, the function $\boldsymbol{u}$ given by (2.13) is a unique solution in $Y^{1,2}(\Omega)^{m}$ of the problem

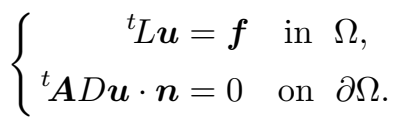

We point out that part iii) in the above definitions give the uniqueness of a Neumann function. Indeed, let $\tilde{\boldsymbol{N}}(x, y)$ be another function satisfying the above properties. Then by uniqueness we have

$$
\int_{\Omega}(\boldsymbol{N}-\tilde{\boldsymbol{N}})(y, x)^{T} \boldsymbol{f}(y) d y=0, \quad \forall \boldsymbol{f} \in C_{c}^{\infty}(\Omega)^{m},
$$

and thus we conclude that $\boldsymbol{N}=\tilde{\boldsymbol{N}}$ a.e. in $\Omega \times \Omega$.

\section{MAin RESUlts}

The following "interior Hölder continuity" condition (IH) means that weak solutions of $L \boldsymbol{u}=0$ and ${ }^{t} L \boldsymbol{u}=0$ enjoy interior Hölder continuity. In the case $m=1$, it is a consequence of the celebrated De Giorgi-Moser-Nash theorem. If $m>1$ and $d>2$, it is not true in general, but, however, if the coefficients of system (1.1) belong to the class of VMO and if $\Omega$ is bounded, then it is known that condition (II) holds in that case; see, e.g., [12, Lemma 5.3].

Condition (IH). There exist $\mu_{0} \in(0,1]$ and $C_{0}>0$ such that for all $x \in \Omega$ and $0<R<d_{x}$, where $d_{x}=\operatorname{dist}(x, \partial \Omega)$, the following holds: If $\boldsymbol{u} \in W^{1,2}\left(B_{R}(x)\right)$ is a weak solution of either $L \boldsymbol{u}=0$ or ${ }^{t} L \boldsymbol{u}=0$ in $B_{R}=B_{R}(x)$, then $\boldsymbol{u}$ is Hölder continuous in $B_{R}$ with the following estimate:

$$
[\boldsymbol{u}]_{C^{\mu_{0}\left(B_{R / 2}\right)}} \leq C_{0} R^{-\mu_{0}}\left(f_{B_{R}}|\boldsymbol{u}|^{2}\right)^{1 / 2},
$$

where $[\boldsymbol{u}]_{C^{\mu_{0}\left(B_{R / 2}\right)}}$ denotes the usual Hölder seminorm.

Theorem 3.1. Let $\Omega \subset \mathbb{R}^{d}(d \geq 3)$ be a bounded Lipschitz domain. Assume condition (IH). Then there exist Neumann functions $\mathbf{N}(x, y)$ of $L$ and $\tilde{\mathbf{N}}(x, y)$ of ${ }^{t} L$ in $\Omega$. We have $\boldsymbol{N}(\cdot, y), \tilde{N}(\cdot, y) \in C_{l o c}^{\mu_{0}}(\Omega \backslash\{y\})$ for all $y \in \Omega$, and the following identity holds:

$$
\tilde{\mathbf{N}}(x, y):=\mathbf{N}(y, x)^{T}, \quad \forall x, y \in \Omega, \quad x \neq y .
$$

Moreover, for any $\boldsymbol{f} \in L^{q}(\Omega)^{m}$ with $q>d / 2$ and $\boldsymbol{g} \in L^{2}(\partial \Omega)^{m}$ satisfying $\int_{\Omega} \boldsymbol{f}+$ $\int_{\partial \Omega} \boldsymbol{g}=0$, the function $\boldsymbol{u}$ given by

$$
\boldsymbol{u}(x):=\int_{\Omega} \boldsymbol{N}(x, y) \boldsymbol{f}(y) d y+\int_{\partial \Omega} \boldsymbol{N}(x, y) \boldsymbol{g}(y) d \sigma(y)
$$

is a unique solution in $\tilde{W}^{1,2}(\Omega)^{m}$ of the problem

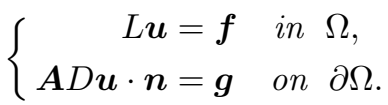


Furthermore, the following estimates hold for all $y \in \Omega$ :

i) $\|\boldsymbol{N}(\cdot, y)\|_{L^{2 d /(d-2)}\left(\Omega \backslash B_{r}(y)\right)}+\|D \boldsymbol{N}(\cdot, y)\|_{L^{2}\left(\Omega \backslash B_{r}(y)\right)} \leq C r^{1-d / 2}$ for all $r \in\left(0, d_{y}\right)$.

ii) $\|\boldsymbol{N}(\cdot, y)\|_{L^{p}\left(B_{r}(y)\right)} \leq C r^{2-d+d / p}$ for all $r \in\left(0, d_{y}\right)$, where $p \in\left[1, \frac{d}{d-2}\right)$.

iii) $|\{x \in \Omega:|\boldsymbol{N}(x, y)|>t\}| \leq C t^{-d /(d-2)}$ for all $t>d_{y}^{2-d}$.

iv) $\|D N(\cdot, y)\|_{L^{p}\left(B_{r}(y)\right)} \leq C r^{1-d+d / p}$ for all $r \in\left(0, d_{y}\right)$, where $p \in\left[1, \frac{d}{d-1}\right)$.

v) $\left|\left\{x \in \Omega:\left|D_{x} \boldsymbol{N}(x, y)\right|>t\right\}\right| \leq C t^{-d /(d-1)}$ for all $t>d_{y}^{1-d}$.

vi) $|\boldsymbol{N}(x, y)| \leq C|x-y|^{2-d}$ whenever $0<|x-y|<d_{y} / 2$.

vii) $\left|\boldsymbol{N}(x, y)-\boldsymbol{N}\left(x^{\prime}, y\right)\right| \leq C\left|x-x^{\prime}\right|^{\mu_{0}}|x-y|^{2-d-\mu_{0}} \quad$ if $x \neq y$ and $2\left|x-x^{\prime}\right|<$ $|x-y|<d_{y} / 2$.

In the above, $C=C\left(d, m, \lambda, M, \Omega, \mu_{0}, C_{0}\right)>0$ and $C$ depends on $p$ as well in ii) and in iv). The estimates $i)$ - vii) are also valid for $\tilde{\mathbf{N}}(x, y)$.

Remark 3.5. Observe that if $\boldsymbol{f} \in L^{q}(\Omega)^{m}$ (where $q>d / 2$ ) satisfies $\int_{\Omega} \boldsymbol{f}=0$, then we may take $\boldsymbol{g}=0$ in (3.3) and conclude that

$$
\boldsymbol{u}(x):=\int_{\Omega} \boldsymbol{N}(x, y) \boldsymbol{f}(y) d y
$$

is a unique solution in $\tilde{W}^{1,2}(\Omega)^{m}$ of the problem

$$
\left\{\begin{aligned}
L \boldsymbol{u}=\boldsymbol{f} & \text { in } \Omega, \\
\boldsymbol{A D u} \cdot \boldsymbol{n}=0 & \text { on } \partial \Omega .
\end{aligned}\right.
$$

Similarly, if $\boldsymbol{g} \in L^{2}(\partial \Omega)^{m}$ satisfies $\int_{\partial \Omega} \boldsymbol{g}=0$, then we find that

$$
\boldsymbol{u}(x):=\int_{\partial \Omega} \boldsymbol{N}(x, y) \boldsymbol{g}(y) d \sigma(y)
$$

is a unique solution in $\tilde{W}^{1,2}(\Omega)^{m}$ of the problem

$$
\left\{\begin{aligned}
L \boldsymbol{u}=0 & \text { in } \Omega, \\
\boldsymbol{A D \boldsymbol { u }} \cdot \boldsymbol{n}=\boldsymbol{g} & \text { on } \partial \Omega .
\end{aligned}\right.
$$

Also, the following estimates are easy consequences of identity (3.2) and the estimates i) - vii) for $\tilde{\mathbf{N}}(\cdot, x)$ :

i) $\|\boldsymbol{N}(x, \cdot)\|_{L^{2 d /(d-2)}\left(\Omega \backslash B_{r}(x)\right)}+\|D \boldsymbol{N}(x, \cdot)\|_{L^{2}\left(\Omega \backslash B_{r}(x)\right)} \leq C r^{1-d / 2}$ for all $r \in$ $\left(0, d_{x}\right)$.

ii) $\|\boldsymbol{N}(x, \cdot)\|_{L^{p}\left(B_{r}(x)\right)} \leq C r^{2-d+d / p}$ for all $r \in\left(0, d_{x}\right)$, where $p \in\left[1, \frac{d}{d-2}\right)$.

iii) $|\{y \in \Omega:|\boldsymbol{N}(x, y)|>t\}| \leq C t^{-d /(d-2)}$ for all $t>d_{x}^{2-d}$.

iv) $\|D N(x, \cdot)\|_{L^{p}\left(B_{r}(x)\right)} \leq C r^{1-d+d / p}$ for all $r \in\left(0, d_{x}\right)$, where $p \in\left[1, \frac{d}{d-1}\right)$.

v) $\left|\left\{y \in \Omega:\left|D_{y} \boldsymbol{N}(x, y)\right|>t\right\}\right| \leq C t^{-d /(d-1)}$ for all $t>d_{x}^{1-d}$.

vi) $|\boldsymbol{N}(x, y)| \leq C|x-y|^{2-d}$ whenever $0<|x-y|<d_{x} / 2$.

vii) $\left|\boldsymbol{N}(x, y)-\boldsymbol{N}\left(x, y^{\prime}\right)\right| \leq C\left|y-y^{\prime}\right|^{\mu_{0}}|x-y|^{2-d-\mu_{0}} \quad$ if $x \neq y$ and $2\left|y-y^{\prime}\right|<$ $|x-y|<d_{x} / 2$.

In particular, we have $|\boldsymbol{N}(x, y)| \leq C|x-y|^{2-d}$ whenever $0<|x-y|<\frac{1}{2} \max \left(d_{x}, d_{y}\right)$.

The following "local boundedness" condition ( $(\mathrm{LB})$ is used to obtain global pointwise bounds for the Neumann function $\boldsymbol{N}(x, y)$ of $L$ in $\Omega$. Again in the case $m=1$, it is well known that condition ( $(\mathrm{LB})$ holds in bounded Lipschitz domains; see, e.g., [18. In the case when $m>1$, this condition does not hold in general and requires certain restrictions on the coefficients and domains. It can be shown, for example, 
that if the coefficients belong to the VMO class and the domain is bounded and has $C^{1}$ boundary, then condition ( $(\underline{\mathrm{LB}})$ holds via $W^{1, p}$ estimates; see the Appendix.

Condition (LB). There exists a constant $C_{1}>0$ such that the following holds: For any $\boldsymbol{f} \in C_{c}^{\infty}(\Omega)^{m}$ and $\boldsymbol{g} \in C^{\infty}(\partial \Omega)^{m}$ satisfying $\int_{\Omega} \boldsymbol{f}+\int_{\partial \Omega} \boldsymbol{g}=0$, let $\boldsymbol{u} \in \tilde{W}^{1,2}(\Omega)^{m}$ be a unique weak solution of the problem

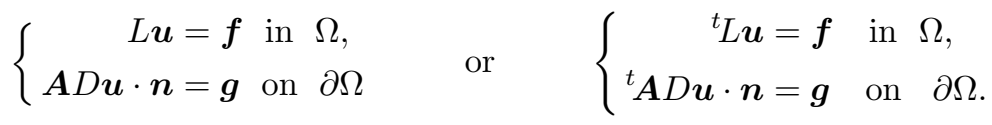

Then for all $x \in \Omega$ and $0<R<\operatorname{diam}(\Omega)$, we have

(LB)

$$
\|\boldsymbol{u}\|_{L^{\infty}\left(\Omega_{R / 2}(x)\right)} \leq C_{1}\left(R^{-d / 2}\|\boldsymbol{u}\|_{L^{2}\left(\Omega_{R}(x)\right)}+R^{2}\|\boldsymbol{f}\|_{L^{\infty}\left(\Omega_{R}(x)\right)}+R\|\boldsymbol{g}\|_{L^{\infty}\left(\Sigma_{R}(x)\right)}\right) .
$$

Theorem 3.6. Let $\Omega \subset \mathbb{R}^{d}(d \geq 3)$ be a bounded Lipschitz domain. Assume condition (IH) and let $\boldsymbol{N}(x, y)$ be the Neumann function of $L$ in $\Omega$ as constructed in Theorem [3.1. If we further assume condition ( $\mathrm{LB}$, then we have the following global pointwise bound for the Neumann function:

$$
|\boldsymbol{N}(x, y)| \leq C|x-y|^{2-d} \quad \text { for all } x, y \in \Omega \text { with } x \neq y,
$$

where $C=C\left(d, m, \lambda, M, \Omega, C_{1}\right)$. Moreover, for all $y \in \Omega$ and $0<r<\operatorname{diam}(\Omega)$, we have

i) $\|\boldsymbol{N}(\cdot, y)\|_{L^{2 d /(d-2)}\left(\Omega \backslash B_{r}(y)\right)}+\|D \boldsymbol{N}(\cdot, y)\|_{L^{2}\left(\Omega \backslash B_{r}(y)\right)} \leq C r^{1-d / 2}$.

ii) $\|\boldsymbol{N}(\cdot, y)\|_{L^{p}\left(B_{r}(y)\right)} \leq C r^{2-d+d / p}$ for $p \in\left[1, \frac{d}{d-2}\right)$.

iii) $|\{x \in \Omega:|\boldsymbol{N}(x, y)|>t\}| \leq C t^{-d /(d-2)}$ for all $t>0$.

iv) $\|D N(\cdot, y)\|_{L^{p}\left(B_{r}(y)\right)} \leq C r^{1-d+d / p}$ for $p \in\left[1, \frac{d}{d-1}\right)$.

v) $\left|\left\{x \in \Omega:\left|D_{x} \boldsymbol{N}(x, y)\right|>t\right\}\right| \leq C t^{-d /(d-1)}$ for all $t>0$.

In the above, $C=C\left(d, m, \lambda, M, \Omega, C_{1}\right)>0$ and $C$ depends on $p$ as well in ii) and in iv). The estimates i) - v) are also valid for the Neumann function $\tilde{\boldsymbol{N}}$ of the adjoint ${ }^{t} L$.

Remark 3.8. As we have pointed out, condition $(\underline{\mathrm{LB}})$ is satisfied, for example, in the scalar case and also in the case when the system has VMO coefficients and the domain is of class $C^{1}$. In fact, in those cases, we also have the following "local Hölder continuity" condition: There exist constants $\mu_{0} \in(0,1]$ and $C_{1}>0$ such that for all $x \in \Omega$ and $0<R<\operatorname{diam}(\Omega)$, the following holds: Let $\boldsymbol{u} \in W^{1,2}\left(\Omega_{R}(x)\right)^{m}$ be a weak solution of either

$$
\begin{array}{rlrl}
L \boldsymbol{u} & =0 \text { in } \Omega_{R}(x), & \boldsymbol{A} D \boldsymbol{u} \cdot \boldsymbol{n}=\boldsymbol{g} \text { on } \Sigma_{R}(x) \\
\text { or } \quad{ }^{t} L \boldsymbol{u} & =0 \text { in } \Omega_{R}(x), \quad{ }^{t} \boldsymbol{A} D \boldsymbol{u} \cdot \boldsymbol{n}=\boldsymbol{g} \text { on } \Sigma_{R}(x),
\end{array}
$$

where $\boldsymbol{g} \in C^{\infty}(\partial \Omega)^{m}$, then we have

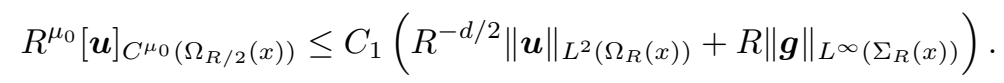

By using $(\overline{\mathrm{LH}})$ and modifying the proof for estimate vii) in Theorem 3.1 (cf. the proof for (3.7) in Section 4.2), we have the following global version of the estimate vii):

$\left|\boldsymbol{N}(x, y)-\boldsymbol{N}\left(x^{\prime}, y\right)\right| \leq C\left|x-x^{\prime}\right|^{\mu_{0}}|x-y|^{2-d-\mu_{0}}$ if $x \neq y$ and $2\left|x-x^{\prime}\right|<|x-y|$, where $C=C\left(d, m, \lambda, M, \Omega, \mu_{0}, C_{1}\right)>0$. The same estimate is also valid for $\tilde{\boldsymbol{N}}$. 
Finally, the following theorem says that the converse of Theorem 3.6 is also true, and thus that condition (LB) is equivalent to a global bound (3.7) for the Neumann function.

Theorem 3.9. Let $\Omega \subset \mathbb{R}^{d}(d \geq 3)$ be a bounded Lipschitz domain. Assume condition (IH) and let $\mathbf{N}(x, y)$ be the Neumann function of $L$ in $\Omega$. Suppose there exists a constant $C_{2}$ such that we have

$$
|\boldsymbol{N}(x, y)| \leq C_{2}|x-y|^{2-d}, \quad \forall x, y \in \Omega, \quad x \neq y .
$$

Then condition (LB) is satisfied in $\Omega$ with $C_{1}=C_{1}\left(d, m, \lambda, M, \Omega, C_{2}\right)$.

\section{Proofs of MAIN THEOREMS}

4.1. Proof of Theorem [3.1, We closely follow the proof of [12, Theorem 3.1]. Let us fix a function $\Phi \in C_{c}^{\infty}\left(\mathbb{R}^{d}\right)$ such that $\Phi$ is supported in $B_{1}(0), 0 \leq \Phi \leq 2$, and $\int_{\mathbb{R}^{d}} \Phi=1$. Let $y \in \Omega$ be fixed but arbitrary. For $\varepsilon>0$, we define

$$
\Phi_{\varepsilon}(x)=\varepsilon^{-d} \Phi((x-y) / \varepsilon) .
$$

Let $\boldsymbol{v}=\boldsymbol{v}_{\varepsilon, y, k}$ be a unique weak solution in $\tilde{W}^{1,2}(\Omega)^{m}$ of the problem (see Section 2.3)

$$
\left\{\begin{aligned}
L \boldsymbol{v} & =\Phi_{\varepsilon} \boldsymbol{e}_{k} \text { in } \Omega \\
\boldsymbol{A D} \boldsymbol{v} \cdot \boldsymbol{n} & =-(1 /|\partial \Omega|) \boldsymbol{e}_{k} \text { on } \partial \Omega
\end{aligned}\right.
$$

where $\boldsymbol{e}_{k}$ is the $k$-th unit vector in $\mathbb{R}^{m}$. We define the "mollified Neumann function" $\boldsymbol{N}^{\varepsilon}(\cdot, y)=\left(N_{j k}^{\varepsilon}(\cdot, y)\right)_{j, k=1}^{m}$ by

$$
N_{j k}^{\varepsilon}(\cdot, y)=v^{j}=v_{\varepsilon, y, k}^{j} .
$$

Then $\boldsymbol{N}^{\varepsilon}(\cdot, y)$ satisfies the following identity (see (2.10) $)$ :

$$
\int_{\Omega} A_{i j}^{\alpha \beta} D_{\beta} N_{j k}^{\varepsilon}(\cdot, y) D_{\alpha} \phi^{i}+\frac{1}{|\partial \Omega|} \int_{\partial \Omega} \phi^{k}=\int_{\Omega_{\varepsilon}(y)} \Phi_{\varepsilon} \phi^{k}, \quad \forall \phi \in W^{1,2}(\Omega)^{m} .
$$

By the definition of the space $\tilde{W}^{1,2}(\Omega)$, we have in particular the following identity:

$$
\int_{\Omega} A_{i j}^{\alpha \beta} D_{\beta} N_{j k}^{\varepsilon}(\cdot, y) D_{\alpha} \phi^{i}=\int_{\Omega_{\varepsilon}(y)} \Phi_{\varepsilon} \phi^{k}, \quad \forall \phi \in \tilde{W}^{1,2}(\Omega)^{m} .
$$

By taking $\phi=v$ in (4.4) and then using (2.3), Hölder's inequality, and (2.8), we get

$$
\lambda\|D \boldsymbol{v}\|_{L^{2}(\Omega)}^{2} \leq\left|\int_{\Omega_{\varepsilon}(y)} \Phi_{\varepsilon} v^{k}\right| \leq C \varepsilon^{(2-d) / 2}\|\boldsymbol{v}\|_{L^{2 d /(d-2)}(\Omega)} \leq C \varepsilon^{(2-d) / 2}\|D \boldsymbol{v}\|_{L^{2}(\Omega)} .
$$

Therefore, we have (recall $\boldsymbol{v}$ is the $k$-th column of $\boldsymbol{N}^{\varepsilon}(\cdot, y)$ )

$$
\left\|D \boldsymbol{N}^{\varepsilon}(\cdot, y)\right\|_{L^{2}(\Omega)} \leq C \varepsilon^{(2-d) / 2}, \quad \text { where } C=C(d, m, \lambda, M, \Omega) .
$$

Let $R \in\left(0, d_{y}\right)$ be arbitrary, but fixed. Assume that $f \in C_{c}^{\infty}(\Omega)^{m}$ is supported in $B_{R}=B_{R}(y) \subset \Omega$. Let $\boldsymbol{u}$ be a unique weak solution in $\tilde{W}^{1,2}(\Omega)^{m}$ of problem (2.14). We then have the following identity (recall ${ }^{t} A_{j i}^{\beta \alpha}=A_{i j}^{\alpha \beta}$ ):

$$
\int_{\Omega} A_{i j}^{\alpha \beta} D_{\beta} w^{j} D_{\alpha} u^{i}=\int_{\Omega} f^{i} w^{i}, \quad \forall \boldsymbol{w} \in \tilde{W}^{1,2}(\Omega)^{m} .
$$


Then by setting $\boldsymbol{\phi}=\boldsymbol{u}$ in (4.4) and setting $\boldsymbol{w}=\boldsymbol{v}_{\varepsilon, y, k}$ in (4.6), we get

$$
\int_{\Omega} N_{i k}^{\varepsilon}(\cdot, y) f^{i}=\int_{\Omega_{\varepsilon}(y)} \Phi_{\varepsilon} u^{k} .
$$

Also, by taking $\boldsymbol{w}=\boldsymbol{u}$ in (4.6) and by using (2.3), (2.8), and Hölder's inequality, we get

$$
\lambda\|D \boldsymbol{u}\|_{L^{2}(\Omega)}^{2} \leq \int_{\Omega} A_{i j}^{\alpha \beta} D_{\beta} u^{j} D_{\alpha} u^{i}=\int_{\Omega} f^{i} u^{i} \leq C\|\boldsymbol{f}\|_{L^{2 d /(d+2)}(\Omega)}\|D \boldsymbol{u}\|_{L^{2}(\Omega)} .
$$

Therefore, we have the estimate

$$
\|D \boldsymbol{u}\|_{L^{2}(\Omega)} \leq C\|\boldsymbol{f}\|_{L^{2 d /(d+2)}(\Omega)} .
$$

We remark that condition $(\mathrm{IH})$ is equivalent to the property $(\mathrm{H})$ in 12 , Definition 2.1]; see [12, Lemma 2.3 and 2.4]. By utilizing condition (IH) and (4.8), and following literally the same steps used in deriving [12, Eq. (3.15)], we obtain

$$
\|\boldsymbol{u}\|_{L^{\infty}\left(B_{R / 2}\right)} \leq C R^{2}\|\boldsymbol{f}\|_{L^{\infty}\left(B_{R}\right)},
$$

where $C=C\left(d, m, \lambda, M, \Omega, \mu_{0}, C_{0}\right)$. Since $R \in\left(0, d_{y}\right)$ is arbitrary, we get from the above estimate and (4.7) that

$\left|\int_{B_{R}} N_{i k}^{\varepsilon}(\cdot, y) f^{i}\right| \leq C R^{2}\|\boldsymbol{f}\|_{L^{\infty}\left(B_{R}\right)}, \quad \forall \boldsymbol{f} \in C_{c}^{\infty}\left(B_{R}\right), \quad \forall \varepsilon \in(0, R / 2), \quad \forall R \in\left(0, d_{y}\right)$.

Therefore, by duality, we conclude that

$$
\left\|\boldsymbol{N}^{\varepsilon}(\cdot, y)\right\|_{L^{1}\left(B_{R}(y)\right)} \leq C R^{2}, \quad \forall \varepsilon \in(0, R / 2), \quad \forall R \in\left(0, d_{y}\right) .
$$

Now, for any $x \in \Omega$ such that $0<|x-y|<d_{y} / 2$, let us take $R:=2|x-y| / 3$. Notice that if $\varepsilon<R / 2$, then $\boldsymbol{N}^{\varepsilon}(\cdot, y) \in W^{1,2}\left(B_{R}(x)\right)^{m^{2}}$ and satisfies $L \boldsymbol{N}^{\varepsilon}(\cdot, y)=$ 0 in $B_{R}(x)$. Then by following the same line of argument used in deriving [12, Eq. (3.19)], for any $x, y \in \Omega$ satisfying $0<|x-y|<d_{y} / 2$, we have

$$
\left|\boldsymbol{N}^{\varepsilon}(x, y)\right| \leq C|x-y|^{2-d}, \quad \forall \varepsilon<|x-y| / 3 .
$$

Next, fix any $r \in\left(0, d_{y} / 2\right)$ and let $\boldsymbol{v}_{\varepsilon}$ be the $k$-th column of $\boldsymbol{N}^{\varepsilon}(\cdot, y)$, where $k=1, \ldots, m$ and $0<\varepsilon<r / 6$. Let $\eta$ be a smooth function on $\mathbb{R}^{d}$ satisfying

$$
0 \leq \eta \leq 1, \quad \eta \equiv 1 \text { on } \mathbb{R}^{d} \backslash B_{r}(y), \quad \eta \equiv 0 \text { on } B_{r / 2}(y), \quad \text { and } \quad|D \eta| \leq 4 / r .
$$

We set $\phi=\eta^{2} \boldsymbol{v}_{\varepsilon}$ in (4.4) and then use (4.9) to obtain

$$
\int_{\Omega} \eta^{2}\left|D \boldsymbol{v}_{\varepsilon}\right|^{2} \leq C \int_{\Omega}|D \eta|^{2}\left|\boldsymbol{v}_{\varepsilon}\right|^{2} \leq C r^{-2} \int_{B_{r}(y) \backslash B_{r / 2}(y)}|x-y|^{2(2-d)} d x \leq C r^{2-d} .
$$

Therefore, by (2.8) and (4.11), we obtain

$$
\left\|\boldsymbol{v}_{\varepsilon}\right\|_{L^{2 d /(d-2)}\left(\Omega \backslash B_{r}(y)\right)} \leq\left\|\eta \boldsymbol{v}_{\varepsilon}\right\|_{L^{2 d /(d-2)}(\Omega)} \leq C\left\|D\left(\eta \boldsymbol{v}_{\varepsilon}\right)\right\|_{L^{2}(\Omega)} \leq C r^{(2-d) / 2},
$$

provided that $0<\varepsilon<r / 6$. On the other hand, if $\varepsilon \geq r / 6$, then (4.5) implies

$$
\left\|\boldsymbol{v}_{\varepsilon}\right\|_{L^{2 d /(d-2)}\left(\Omega \backslash B_{r}(y)\right)} \leq\left\|\boldsymbol{v}_{\varepsilon}\right\|_{L^{2 d /(d-2)}(\Omega)} \leq C\left\|D \boldsymbol{v}_{\varepsilon}\right\|_{L^{2}(\Omega)} \leq C r^{(2-d) / 2} .
$$

By combining the above two estimates, we obtain

$$
\left\|\boldsymbol{N}^{\varepsilon}(\cdot, y)\right\|_{L^{2 d /(d-2)}\left(\Omega \backslash B_{r}(y)\right)} \leq C r^{(2-d) / 2}, \quad \forall r \in\left(0, d_{y} / 2\right), \quad \forall \varepsilon>0 .
$$


Notice from (4.11) and (4.10) that for $0<\varepsilon<r / 6$, we have

$$
\left\|D \boldsymbol{N}^{\varepsilon}(\cdot, y)\right\|_{L^{2}\left(\Omega \backslash B_{r}(y)\right)} \leq C r^{(2-d) / 2} .
$$

In the case when $\varepsilon \geq r / 6$, we obtain from (4.5) that

$$
\left\|D \boldsymbol{N}^{\varepsilon}(\cdot, y)\right\|_{L^{2}\left(\Omega \backslash B_{r}(y)\right)} \leq\left\|D \boldsymbol{N}^{\varepsilon}(\cdot, y)\right\|_{L^{2}(\Omega)} \leq C \varepsilon^{(2-d) / 2} \leq C r^{(2-d) / 2} .
$$

By combining the above two inequalities, we obtain

$$
\left\|D \boldsymbol{N}^{\varepsilon}(\cdot, y)\right\|_{L^{2}\left(\Omega \backslash B_{r}(y)\right)} \leq C r^{(2-d) / 2}, \quad \forall r \in\left(0, d_{y} / 2\right), \quad \forall \varepsilon>0 .
$$

From the the obvious fact that $d_{y} / 2$ and $d_{y}$ are comparable to each other, we find by (4.12) and (4.13) that

$$
\begin{array}{r}
\left\|\boldsymbol{N}^{\varepsilon}(\cdot, y)\right\|_{L^{2 d /(d-2)}\left(\Omega \backslash B_{r}(y)\right)}+\left\|D \boldsymbol{N}^{\varepsilon}(\cdot, y)\right\|_{L^{2}\left(\Omega \backslash B_{r}(y)\right)} \leq C r^{(2-d) / 2}, \\
\forall r \in\left(0, d_{y}\right), \quad \forall \varepsilon>0 .
\end{array}
$$

From (4.14) it follows that (see [12, pp. 147-148])

$$
\begin{aligned}
\left|\left\{x \in \Omega:\left|\boldsymbol{N}^{\varepsilon}(x, y)\right|>t\right\}\right| & \leq C t^{-d /(d-2)}, & \forall t>d_{y}^{2-d}, & \forall \varepsilon>0, \\
\left|\left\{x \in \Omega:\left|D_{x} \boldsymbol{N}^{\varepsilon}(x, y)\right|>t\right\}\right| & \leq C t^{-d /(d-1)}, & \forall t>d_{y}^{1-d}, & \forall \varepsilon>0 .
\end{aligned}
$$

It is routine to derive the following strong type estimates from the above weak type estimates (4.15) and (4.16) (see e.g. [12, p. 148]):

$$
\left\|\boldsymbol{N}^{\varepsilon}(\cdot, y)\right\|_{L^{p}\left(B_{r}(y)\right)} \leq C r^{2-d+d / p}, \quad \forall r \in\left(0, d_{y}\right), \quad \forall \varepsilon>0, \text { for } 1 \leq p<d /(d-2),
$$

$$
\left\|D \boldsymbol{N}^{\varepsilon}(\cdot, y)\right\|_{L^{p}\left(B_{r}(y)\right)} \leq C r^{1-d+d / p}, \quad \forall r \in\left(0, d_{y}\right), \quad \forall \varepsilon>0, \text { for } 1 \leq p<d /(d-1),
$$

where $C=C\left(d, m, \lambda, M, \Omega, \mu_{0}, C_{0}, p\right)$.

From (4.13), (4.17), and (4.18), it follows that that there exists a sequence $\left\{\varepsilon_{\mu}\right\}_{\mu=1}^{\infty}$ tending to zero and a function $\boldsymbol{N}(\cdot, y)$ such that $\boldsymbol{N}^{\varepsilon_{\mu}}(\cdot, y) \rightarrow \boldsymbol{N}(\cdot, y)$ weakly in $W^{1, p}\left(B_{r}(y)\right)$ for $1<p<d /(d-1)$ and all $r \in\left(0, d_{y}\right)$ and also that $\boldsymbol{N}^{\varepsilon_{\mu}}(\cdot, y) \rightarrow \boldsymbol{N}(\cdot, y)$ weakly in $W^{1,2}\left(\Omega \backslash B_{r}(y)\right)$ for all $r \in\left(0, d_{y}\right)$; see [12, p. 159] for the details. Then it is routine to check that $\boldsymbol{N}(\cdot, y)$ satisfies properties i) and ii) in Section 2.4.1, and also the estimates i) - v) in the theorem; see [12, Section 4.1].

We now turn to the pointwise bound for $\boldsymbol{N}(x, y)$. For any $x \in \Omega$ such that $0<|x-y|<d_{y} / 2$, set $R:=2|x-y| / 3$. Notice that (4.14) implies that $\boldsymbol{N}(\cdot, y) \in$ $W^{1,2}\left(B_{R}(x)\right)$ and satisfies $L \boldsymbol{N}(\cdot, y)=0$ weakly in $B_{R}(x)$. Then, by [12, Lemma 2.4] and estimate ii) in the theorem, we have

$$
\begin{aligned}
|\boldsymbol{N}(x, y)| & \leq C R^{-d}\|\boldsymbol{N}(\cdot, y)\|_{L^{1}\left(B_{R}(x)\right)} \leq C R^{-d}\|\boldsymbol{N}(\cdot, y)\|_{L^{1}\left(B_{3 R}(y)\right)} \\
& \leq C R^{2-d} \leq C|x-y|^{2-d} .
\end{aligned}
$$

We have thus shown that estimate vi) in the theorem holds. Then, it is routine to see that estimate vii) in the theorem follows from condition (IH) and the above estimate.

Next, let $x \in \Omega \backslash\{y\}$ be fixed but arbitrary, and let $\tilde{\boldsymbol{N}}^{\varepsilon^{\prime}}(\cdot, x) \in \tilde{W}^{1,2}(\Omega)^{m^{2}}$ be the mollified Neumann function of the adjoint operator ${ }^{t} L$ in $\Omega$, where $\varepsilon^{\prime}>0$. 
By setting $\phi$ in (4.4) to be the $l$-th column of $\tilde{\boldsymbol{N}}^{\varepsilon^{\prime}}(\cdot, x)$ and utilizing an integral identity for $\tilde{N}^{\varepsilon^{\prime}}(\cdot, x)$ similar to (4.4), we obtain the following identity:

$$
\int_{\Omega_{\varepsilon^{\prime}}(x)} \Phi_{\varepsilon^{\prime}} N_{l k}^{\varepsilon}(\cdot, y)=\int_{\Omega_{\varepsilon}(y)} \Phi_{\varepsilon} \tilde{N}_{k l}^{\varepsilon^{\prime}}(\cdot, x) .
$$

Let $\tilde{\boldsymbol{N}}(\cdot, x)$ be a Neumann function of ${ }^{t} L$ in $\Omega$ obtained by a sequence $\left\{\varepsilon_{\nu}^{\prime}\right\}_{\nu=1}^{\infty}$ tending to 0 . Then, by following the same steps as in [12, p. 151], we conclude

$$
N_{l k}(x, y)=\tilde{N}_{k l}(y, x), \quad \forall k, l=1, \ldots, m,
$$

which obviously implies the identity (3.2). In fact, by following a similar line of reasoning as in [12, p. 151], we find

$$
\begin{aligned}
\boldsymbol{N}^{\varepsilon}(x, y) & =\varepsilon^{-d} \int_{\Omega} \Phi\left(\frac{z-y}{\varepsilon}\right) \boldsymbol{N}(x, z) d z, \\
\lim _{\varepsilon \rightarrow 0} \boldsymbol{N}^{\varepsilon}(x, y) & =\boldsymbol{N}(x, y), \quad \forall x, y \in \Omega, \quad x \neq y .
\end{aligned}
$$

Now, let $\boldsymbol{u}$ be a unique solution in $\tilde{W}^{1,2}(\Omega)^{m}$ of problem (2.14) with $\boldsymbol{f} \in$ $C_{c}^{\infty}(\Omega)^{m}$. We remark that condition (IH) implies that $\boldsymbol{u}$ is continuous in $\Omega$; see [12, Eq. (3.14)]. By setting $\boldsymbol{w}$ to be the $k$-th column of $\boldsymbol{N}^{\varepsilon}(\cdot, y)$ in (4.6) and setting $\phi=\boldsymbol{u}$ in (4.4), we get

$$
\int_{\Omega} N_{i k}^{\varepsilon}(\cdot, y) f^{i}=\int_{\Omega_{\varepsilon}(y)} \Phi_{\varepsilon} u^{k}
$$

We take the limit $\varepsilon \rightarrow 0$ above to get

$$
u^{k}(y)=\int_{\Omega} N_{i k}(x, y) f^{i}(x) d x,
$$

which is equivalent to (2.13). We have shown that $\boldsymbol{N}(x, y)$ satisfies property iii) in Section 2.4.1 and thus that $\boldsymbol{N}(x, y)$ is a unique Neumann function of the operator $L$ in $\Omega$.

Finally, let $\boldsymbol{f} \in L^{q}(\Omega)^{m}$ with $q>d / 2$ and $\boldsymbol{g} \in L^{2}(\partial \Omega)^{m}$ satisfy the compatibility condition (2.9), and let $\boldsymbol{u}$ be a unique weak solution in $\tilde{W}^{1,2}(\Omega)^{m}$ of problem (3.4); see Section 2.3. Then $\boldsymbol{u}$ satisfies identity (2.10). By setting $\boldsymbol{v}$ to be the $k$-th column of $\tilde{\boldsymbol{N}}^{\varepsilon}(\cdot, x)$ in (2.10) and utilizing an integral identity for $\tilde{\boldsymbol{N}}^{\varepsilon}(\cdot, x)$ similar to (4.4), we get

$$
\int_{\Omega} \tilde{N}_{i k}^{\varepsilon}(\cdot, x) f^{i}+\int_{\partial \Omega} \tilde{N}_{i k}^{\varepsilon}(\cdot, x) g^{i}=\int_{\Omega_{\varepsilon}(x)} \Phi_{\varepsilon} u^{k}
$$

We remark that condition $(\overline{\mathrm{IH}})$ together with the assumption that $f \in L^{q}(\Omega)^{m}$ with $q>d / 2$ implies that $\boldsymbol{u}$ is Hölder continuous in $\Omega$; see, e.g., [12, Section 3.2]. Then by proceeding similarly as above and using (3.2), we obtain

$$
u^{k}(x)=\int_{\Omega} N_{k i}(x, y) f^{i}(y) d y+\int_{\Omega} N_{k i}(x, y) g^{i}(y) d \sigma(y),
$$

which is formula (3.3). The proof is complete. 
4.2. Proof of Theorem 3.6. First, we shall assume that $0<|x-y| \leq 2 / 3$ and prove the bound (3.7). Let $0<R<1$ and $y \in \Omega$ be arbitrary, but fixed. Assume that $\boldsymbol{f} \in C_{c}^{\infty}(\Omega)^{m}$ is supported in $\Omega_{R}(y)$ and let $\boldsymbol{u}$ be a unique weak solution in $\tilde{W}^{1,2}(\Omega)^{m}$ of problem (2.14). Then we have the identities (4.6) and (4.7) as in the proof of Theorem 3.1. Also, we have the estimate (4.8), and thus by (2.8) we get $(4.21)$

$$
\|\boldsymbol{u}\|_{L^{2 d /(d-2)}(\Omega)} \leq C\|D \boldsymbol{u}\|_{L^{2}(\Omega)} \leq C\|\boldsymbol{f}\|_{L^{2 d /(d+2)}(\Omega)} \leq C R^{(2+d) / 2}\|\boldsymbol{f}\|_{L^{\infty}\left(\Omega_{R}(y)\right)},
$$

where $C=C(d, m, \lambda, M, \Omega)$. Observe that $\boldsymbol{g}:=-(1 /|\partial \Omega|) \int_{\Omega} \boldsymbol{f}$ has the bound

$$
|\boldsymbol{g}| \leq \frac{1}{|\partial \Omega|} \int_{\Omega}|\boldsymbol{f}| \leq C R^{d}\|\boldsymbol{f}\|_{L^{\infty}\left(\Omega_{R}(y)\right)}
$$

where $C=C(d, \Omega)$, and thus we have

$$
R\|\boldsymbol{g}\|_{L^{\infty}\left(\Sigma_{R}(y)\right)} \leq C R^{d+1}\|\boldsymbol{f}\|_{L^{\infty}\left(\Omega_{R}(y)\right)} \leq C R^{2}\|\boldsymbol{f}\|_{L^{\infty}\left(\Omega_{R}(y)\right)},
$$

where we used the assumption that $R<1$. Then by (LB), (4.21), (4.22), and Hölder's inequality, we obtain

$$
\|\boldsymbol{u}\|_{L^{\infty}\left(\Omega_{R / 2}(y)\right)} \leq C R^{2}\|\boldsymbol{f}\|_{L^{\infty}\left(\Omega_{R}(y)\right)},
$$

where $C=C\left(d, m, \lambda, M, \Omega, C_{1}\right)$. Hence, by (4.7) and (4.23), we conclude that

$$
\left|\int_{\Omega_{R}(y)} \boldsymbol{N}^{\varepsilon}(\cdot, y)^{T} \boldsymbol{f}\right| \leq C R^{2}\|\boldsymbol{f}\|_{L^{\infty}\left(\Omega_{R}(y)\right)}, \quad \forall \boldsymbol{f} \in C_{c}^{\infty}\left(\Omega_{R}(y)\right), \quad \forall \varepsilon \in(0, R / 2) .
$$

Therefore, by duality, we conclude from (4.24) that

$$
\left\|\boldsymbol{N}^{\varepsilon}(\cdot, y)\right\|_{L^{1}\left(\Omega_{R}(y)\right)} \leq C R^{2}, \quad \forall \varepsilon \in(0, R / 2),
$$

where $C=C\left(d, m, \lambda, M, \Omega, C_{1}\right)$.

Next, recall that $\boldsymbol{v}=\boldsymbol{v}_{\varepsilon, y, k}$ (i.e., the $k$-th column of $\boldsymbol{N}^{\varepsilon}(\cdot, y)$ ) is a unique weak solution in $\tilde{W}^{1,2}(\Omega)^{m}$ of problem (4.1). Let $x \in \Omega, r>0$, and $\varepsilon>0$ be such that $B_{\varepsilon}(y) \cap B_{r}(x)=\emptyset$. Then, condition (LB) implies that

$$
\left\|\boldsymbol{N}^{\varepsilon}(\cdot, y)\right\|_{L^{\infty}\left(\Omega_{r / 2}(x)\right)} \leq C_{1}\left(r^{-d / 2}\left\|\boldsymbol{N}^{\varepsilon}(\cdot, y)\right\|_{L^{2}\left(\Omega_{r}(x)\right)}+|\partial \Omega|^{-1} r\right) .
$$

By a standard iteration argument (see [10, pp. 80-82]), we then obtain from (4.26) that

$$
\left\|\boldsymbol{N}^{\varepsilon}(\cdot, y)\right\|_{L^{\infty}\left(\Omega_{r / 2}(x)\right)} \leq C r^{-d}\left\|\boldsymbol{N}^{\varepsilon}(\cdot, y)\right\|_{L^{1}\left(\Omega_{r}(x)\right)}+C r,
$$

where $C=C\left(d, C_{1},|\partial \Omega|\right)$.

Now, for any $x \in \Omega$ satisfying $0<|x-y| \leq 2 / 3$, take $R=3 r=3|x-y| / 2$. Then by (4.27) and (4.25), we obtain for all $\varepsilon \in(0, r)$ that

$$
\begin{array}{r}
\left|\boldsymbol{N}^{\varepsilon}(x, y)\right| \leq C r^{-d}\left\|\boldsymbol{N}^{\varepsilon}(\cdot, y)\right\|_{L^{1}\left(\Omega_{r}(x)\right)}+C r \leq C r^{-d}\left\|\boldsymbol{N}^{\varepsilon}(\cdot, y)\right\|_{L^{1}\left(\Omega_{3 r}(y)\right)}+C r \\
\leq C R^{2-d}+C R \leq C R^{2-d} \leq C|x-y|^{2-d},
\end{array}
$$

where $C=C\left(d, m, \lambda, M, \Omega, C_{1}\right)$, and we have again used the assumption that $R \leq 1$. Therefore, by using (4.20), we may take the limit $\varepsilon \rightarrow 0$ in the above inequality and obtain (3.7) under an extra assumption that $|x-y| \leq 2 / 3$. In the case when $|x-y|>2 / 3$, we take $R=3 r=1$ in (4.28) and get

$$
\left|\boldsymbol{N}^{\varepsilon}(x, y)\right| \leq C \leq C \operatorname{diam}(\Omega)^{d-2}|x-y|^{2-d} \leq C|x-y|^{2-d},
$$


where $C=C\left(d, m, \lambda, M, \Omega, C_{1}\right)$. Again, by taking the limit $\varepsilon \rightarrow 0$ in the above inequality, we obtain (3.7) even if $|x-y|>2 / 3$. We have thus shown that (LB) implies (3.7).

To derive the estimates i) - v) in the theorem, we need to repeat some steps in the proof of Theorem 3.1 with a little modification. Let $\boldsymbol{v}_{\varepsilon}$ be the $k$-th column of $\boldsymbol{N}^{\varepsilon}(\cdot, y)$, where $k=1, \ldots, m, 0<\varepsilon<\min \left(d_{y}, r\right) / 6$, and $0<r<\operatorname{diam}(\Omega)$. Let $\eta$ be a smooth function on $\mathbb{R}^{d}$ satisfying conditions (4.10). We set $\boldsymbol{\phi}=\eta^{2} \boldsymbol{v}_{\varepsilon}$ in (4.3) to get

$$
\int_{\Omega} \eta^{2} A_{i j}^{\alpha \beta} D_{\beta} v_{\varepsilon}^{j} D_{\alpha} v_{\varepsilon}^{i}+\int_{\Omega} 2 \eta A_{i j}^{\alpha \beta} D_{\beta} v_{\varepsilon}^{j} D_{\alpha} \eta v_{\varepsilon}^{i}+\frac{1}{|\partial \Omega|} \int_{\partial \Omega}\left(\eta^{2}-1\right) v_{\varepsilon}^{k}=0
$$

where we used the fact $\int_{\partial \Omega} \boldsymbol{v}_{\varepsilon}=0$ and $\eta^{2} \Phi_{\varepsilon} \equiv 0$. We then use (2.3), (2.4), and Cauchy's inequality to get

$$
\int_{\Omega} \eta^{2}\left|D \boldsymbol{N}^{\varepsilon}(\cdot, y)\right|^{2} \leq C\left(\int_{\Omega}|D \eta|^{2}\left|\boldsymbol{N}^{\varepsilon}(\cdot, y)\right|^{2}+\frac{1}{|\partial \Omega|} \int_{\partial \Omega}\left(1-\eta^{2}\right)\left|\boldsymbol{N}^{\varepsilon}(\cdot, y)\right|\right),
$$

where $C=C(\lambda, M)$. By using the conditions in (4.10) and the pointwise bound for $\boldsymbol{N}^{\varepsilon}(x, y)$ obtained above, we get

$$
\begin{aligned}
& \int_{\Omega \backslash B_{r}(y)}\left|D \boldsymbol{N}^{\varepsilon}(\cdot, y)\right|^{2} \\
& \quad \leq C\left(r^{-2} \int_{B_{r}(y) \backslash B_{r / 2}(y)}|x-y|^{4-2 d} d x+\frac{1}{|\partial \Omega|} \int_{\Sigma_{r}(y)}|x-y|^{2-d} d \sigma(x)\right) \\
& \leq C r^{2-d}, \quad \text { where } C=C\left(d, m, \lambda, M, \Omega, C_{1}\right) .
\end{aligned}
$$

Therefore, by taking the limit $\varepsilon \rightarrow 0$, we get

$$
\|D N(\cdot, y)\|_{L^{2}\left(\Omega \backslash B_{r}(y)\right)} \leq C r^{(2-d) / 2}, \quad 0<\forall r<\operatorname{diam}(\Omega) .
$$

Observe that the pointwise bound (3.7) together with the above estimate yields

$$
\begin{aligned}
\|\boldsymbol{N}(\cdot, y)\|_{L^{2 d /(d-2)}\left(\Omega \backslash B_{r}(y)\right)}+\|D N(\cdot, y)\|_{L^{2}\left(\Omega \backslash B_{r}(y)\right)} \leq & C r^{(2-d) / 2}, \\
& 0<\forall r<\operatorname{diam}(\Omega),
\end{aligned}
$$

where $C=C\left(d, m, \lambda, M, \Omega, C_{1}\right)$. By literally following the same step used in deriving (4.15) - (4.18) from (4.14), and using the fact that $|\Omega|<\infty$, we obtain the estimates i) - v) from (4.30). The proof is complete.

4.3. Proof of Theorem 3.9. By symmetry, it is enough to prove (LB) for weak solutions of the problem

$$
\left\{\begin{aligned}
{ }^{t} L \boldsymbol{u}=\boldsymbol{f} & \text { in } \Omega, \\
{ }^{t} \boldsymbol{A} D \boldsymbol{u} \cdot \boldsymbol{n}=\boldsymbol{g} & \text { on } \partial \Omega,
\end{aligned}\right.
$$

where $\boldsymbol{f} \in C_{c}^{\infty}(\Omega)^{m}$ and $\boldsymbol{g} \in C^{\infty}(\partial \Omega)^{m}$ are such that $\int_{\Omega} \boldsymbol{f}+\int_{\partial \Omega} \boldsymbol{g}=0$.

Let $\boldsymbol{u}$ be a unique weak solution in $\tilde{W}^{1,2}(\Omega)^{m}$ of the above problem. We then have the identity (cf. (2.10)

$$
\int_{\Omega} A_{i j}^{\alpha \beta} D_{\beta} w^{j} D_{\alpha} u^{i}=\int_{\partial \Omega} g^{i} w^{i}+\int_{\Omega} f^{i} w^{i}, \quad \forall \boldsymbol{w} \in W^{1,2}(\Omega)^{m} .
$$


Let $\zeta$ be a smooth function on $\mathbb{R}^{d}$ satisfying

$0 \leq \zeta \leq 1, \quad \operatorname{supp} \zeta \subset B_{R / 2}(x), \quad \zeta \equiv 1$ on $B_{3 R / 8}(x), \quad$ and $\quad|D \zeta| \leq 16 / R$.

We set $\boldsymbol{w}=\zeta \boldsymbol{v}_{\varepsilon}$ in (4.31), where $\boldsymbol{v}_{\varepsilon}=\boldsymbol{v}_{\varepsilon, y, k}$ is the $k$-th column of $\boldsymbol{N}^{\varepsilon}(\cdot, y)$, to get

$$
\int_{\Omega} \zeta A_{i j}^{\alpha \beta} D_{\beta} v_{\varepsilon}^{j} D_{\alpha} u^{i}=-\int_{\Omega} A_{i j}^{\alpha \beta} D_{\beta} \zeta v_{\varepsilon}^{j} D_{\alpha} u^{i}+\int_{\partial \Omega} \zeta g^{i} v_{\varepsilon}^{i}+\int_{\Omega} \zeta f^{i} v_{\varepsilon}^{i} .
$$

On the other hand, by setting $\phi=\zeta \boldsymbol{u}$ in (4.3), we get

$$
\int_{\Omega_{\varepsilon}(y)} \Phi_{\varepsilon} \zeta u^{k}=\int_{\Omega} \zeta A_{i j}^{\alpha \beta} D_{\beta} v_{\varepsilon}^{j} D_{\alpha} u^{i}+\int_{\Omega} A_{i j}^{\alpha \beta} D_{\beta} v_{\varepsilon}^{j} D_{\alpha} \zeta u^{i}+\frac{1}{|\partial \Omega|} \int_{\partial \Omega} \zeta u^{k} .
$$

Therefore, by combining (4.33) and (4.34), we obtain

$$
\begin{aligned}
\int_{\Omega_{\varepsilon}(y)} \Phi_{\varepsilon} \zeta u^{k}=\int_{\Omega} A_{i j}^{\alpha \beta} D_{\beta} v_{\varepsilon}^{j} D_{\alpha} \zeta u^{i}+\frac{1}{|\partial \Omega|} \int_{\partial \Omega} \zeta u^{k}-\int_{\Omega} A_{i j}^{\alpha \beta} D_{\beta} \zeta v_{\varepsilon}^{j} D_{\alpha} u^{i} & -\int_{\partial \Omega} \zeta g^{i} v_{\varepsilon}^{i}+\int_{\Omega} \zeta f^{i} v_{\varepsilon}^{i} .
\end{aligned}
$$

Now, assume that $y \in \Omega_{R / 4}(x)$. Notice from (4.32) that $\operatorname{dist}(y, \operatorname{supp} D \zeta)>R / 8$. Then by taking $\varepsilon \rightarrow 0$ in the above identity, we get

$$
\begin{array}{r}
u^{k}(y)=\int_{\Omega} A_{i j}^{\alpha \beta} D_{\beta} N_{j k}(\cdot, y) D_{\alpha} \zeta u^{i}+\frac{1}{|\partial \Omega|} \int_{\partial \Omega} \zeta u^{k}-\int_{\Omega} A_{i j}^{\alpha \beta} D_{\beta} \zeta N_{j k}(\cdot, y) D_{\alpha} u^{i} \\
-\int_{\partial \Omega} \zeta g^{i} N_{i k}(\cdot, y)+\int_{\Omega} \zeta f^{i} N_{j k}(\cdot, y)=: I_{1}+I_{2}+I_{3}+I_{4}+I_{5} .
\end{array}
$$

On the other hand, observe that $\eta^{2} \boldsymbol{v}$, where $\boldsymbol{v}$ is the $k$-th column of $\boldsymbol{N}(\cdot, y)$ and $\eta$ satisfies the properties in (4.10), belongs to $W^{1,2}(\Omega)^{m}$. Then by approximation we may take $\phi=\eta^{2} \boldsymbol{v}$ in (2.12) to get

$$
\int_{\Omega} \eta^{2} A_{i j}^{\alpha \beta} D_{\beta} v^{j} D_{\alpha} v^{i}+\int_{\Omega} 2 \eta A_{i j}^{\alpha \beta} D_{\beta} v^{j} D_{\alpha} \eta v^{i}+\frac{1}{|\partial \Omega|} \int_{\partial \Omega}\left(\eta^{2}-1\right) v^{k}=0,
$$

which corresponds to 4.29) in the proof of Theorem 3.6. Following exactly the same steps as in the proof of Theorem [3.6, we then obtain the estimate (4.30). Also, from (4.31) and the trace theorem, we derive Caccioppoli's inequality

$$
\begin{aligned}
\|D \boldsymbol{u}\|_{L^{2}\left(\Omega_{R / 2}(x)\right)} \leq & C R^{-1}\|\boldsymbol{u}\|_{L^{2}\left(\Omega_{R}(x)\right)}+C R^{d / 2}(1+R)\|\boldsymbol{g}\|_{L^{\infty}\left(\Sigma_{R}(x)\right)} \\
& +C R^{d / 2+1}\|\boldsymbol{f}\|_{L^{\infty}\left(\Omega_{R}(x)\right)},
\end{aligned}
$$

where $C=C(d, m, \lambda, M, \Omega)$; see the Appendix for the proof.

Denote $A_{R}(y)=\Omega_{3 R / 4}(y) \backslash B_{R / 8}(y)$. By using Hölder's inequality, (4.30), and (3.10), we estimate

$$
\begin{aligned}
& \left|I_{1}\right| \leq C R^{-1}\|D N(\cdot, y)\|_{L^{2}\left(A_{R}(y)\right)}\|\boldsymbol{u}\|_{L^{2}\left(\Omega_{R / 2}(x)\right)} \leq C R^{-d / 2}\|\boldsymbol{u}\|_{L^{2}\left(\Omega_{R}(x)\right)} \\
& \left|I_{4}\right| \leq C\|\boldsymbol{N}(\cdot, y)\|_{L^{1}\left(\Sigma_{3 R / 4}(y)\right)}\|\boldsymbol{g}\|_{L^{\infty}\left(\Sigma_{R / 2}(x)\right)} \leq C R\|\boldsymbol{g}\|_{L^{\infty}\left(\Sigma_{R}(x)\right)} \\
& \left|I_{5}\right| \leq C\|\boldsymbol{N}(\cdot, y)\|_{L^{1}\left(\Omega_{3 R / 4}(y)\right)}\|\boldsymbol{f}\|_{L^{\infty}\left(\Omega_{R / 2}(x)\right)} \leq C R^{2}\|\boldsymbol{f}\|_{L^{\infty}\left(\Omega_{R}(x)\right)}
\end{aligned}
$$


Similarly, by Hölder's inequality, the trace theorem, and (4.36), we estimate

$$
\begin{aligned}
\left|I_{2}\right| \leq|\partial \Omega|^{-1 / 2}\|\zeta \boldsymbol{u}\|_{L^{2}(\partial \Omega)} \leq C\left(1+R^{-1}\right)\|\boldsymbol{u}\|_{L^{2}\left(\Omega_{R}(x)\right)}+C\|D \boldsymbol{u}\|_{L^{2}\left(\Omega_{R / 2}(x)\right)} \\
\leq C\left(1+R^{-1}\right)\|\boldsymbol{u}\|_{L^{2}\left(\Omega_{R}(x)\right)}+C\left(R^{d / 2}+R^{d / 2+1}\right)\|\boldsymbol{g}\|_{L^{\infty}\left(\Sigma_{R}(x)\right)} \\
\quad+C R^{d / 2+1}\|\boldsymbol{f}\|_{L^{\infty}\left(\Omega_{R}(x)\right)} .
\end{aligned}
$$

Also, by Hölder's inequality, (4.30), and (4.36), we get

$$
\begin{gathered}
\left|I_{3}\right| \leq C R^{-1}\|\boldsymbol{N}(\cdot, y)\|_{L^{2}\left(A_{R}(y)\right)}\|D \boldsymbol{u}\|_{L^{2}\left(\Omega_{R / 2}(x)\right)} \leq C R^{1-d / 2}\|D \boldsymbol{u}\|_{L^{2}\left(\Omega_{R / 2}(x)\right)} \\
\leq C R^{-d / 2}\|\boldsymbol{u}\|_{L^{2}\left(\Omega_{R}(x)\right)}+C R(1+R)\|\boldsymbol{g}\|_{L^{\infty}\left(\Sigma_{R}(x)\right)}+R^{2}\|\boldsymbol{f}\|_{L^{\infty}\left(\Omega_{R}(x)\right)} .
\end{gathered}
$$

Combining these together, we get from (4.35) that

$$
\begin{aligned}
\|\boldsymbol{u}\|_{L^{\infty}\left(\Omega_{R / 4}\right)} \leq & C R^{-d / 2}\left(1+R^{d / 2}+R^{d / 2-1}\right)\|\boldsymbol{u}\|_{L^{2}\left(\Omega_{R}\right)} \\
& +C R\left(1+R^{d / 2-1}+R^{d / 2}+R\right)\|\boldsymbol{g}\|_{L^{\infty}\left(\Sigma_{R}\right)} \\
& +C R^{2}\left(1+R^{d / 2-1}\right)\|\boldsymbol{f}\|_{L^{\infty}\left(\Omega_{R}(x)\right)}, \quad \text { where } C=C(d, m, \lambda, M, \Omega) .
\end{aligned}
$$

By a standard covering argument and the fact that $R<\operatorname{diam}(\Omega)<\infty$, we obtain (LB) from the above inequality. The proof is complete.

\section{NEUMANN FUNCTIONS IN LIPSCHITZ GRAPH DOMAIN}

This section is devoted to the study of Neumann functions in an unbounded domain above a Lipschitz graph.

5.1. Main results. Since Lipschitz graph domains are necessarily unbounded domains, it is more practical to replace condition $(\overline{\mathrm{IH}})$ in Section 3 by the following condition $\left(\underline{\mathrm{IH}^{\prime}}\right)$. In the case when the domain is bounded, it is equivalent to condition (III), but it is weaker if the domain is unbounded. By the well-known De Giorgi-Moser-Nash theorem, we have condition ( $\underline{\mathrm{IH}^{\prime}}$ with $R_{c}=\infty$ in the scalar case, and thus it reduces to condition (IH).

Condition $\left(\mathbf{I H}^{\prime}\right)$. There exist $\mu_{0} \in(0,1], R_{c} \in(0, \infty]$, and $C_{0}>0$ such that for all $x \in \Omega$ and $R \in\left(0, d_{x}^{\prime}\right)$, where $d_{x}^{\prime}:=\min \left(d_{x}, R_{c}\right)$, the following holds: If $\boldsymbol{u} \in W^{1,2}\left(B_{R}(x)\right)$ is a weak solution of either $L \boldsymbol{u}=0$ or ${ }^{t} L \boldsymbol{u}=0$ in $B_{R}=B_{R}(x)$, then $\boldsymbol{u}$ is Hölder continuous in $B_{R}$ with the following estimate:

$$
[\boldsymbol{u}]_{C^{\mu_{0}\left(B_{R / 2}\right)}} \leq C_{0} R^{-\mu_{0}}\left(f_{B_{R}}|\boldsymbol{u}|^{2}\right)^{1 / 2} .
$$

Theorem 5.1. Let $\Omega$ be a Lipschitz graph domain in $\mathbb{R}^{d}(d \geq 3)$. Assume condition $\left(\underline{\mathrm{IH}^{\prime}}\right)$. Then there exist Neumann functions $\mathbf{N}(x, y)$ of $L$ and $\tilde{\mathbf{N}}(x, y)$ of ${ }^{t} L$ in $\Omega$ satisfying identity (3.2). Furthermore, estimates i) - vii) in Theorem 3.1 are valid for $\boldsymbol{N}(\cdot, y)$ and $\tilde{\mathbf{N}}(\cdot, y)$ for all $y \in \Omega$, provided $d_{x}$ is replaced by $d_{x}^{\prime}=\min \left(d_{x}, R_{c}\right)$.

We also replace condition (LB) in Section 3 by the following condition $\left(\underline{\mathrm{LB}^{\prime}}\right)$. In the scalar case, it is well known that condition ( $\mathrm{LB}^{\prime}$ holds in Lipschitz graph domains.

Condition $\left(\mathbf{L B}^{\prime}\right)$. There exists a constant $C_{1}>0$ such that the following holds: For any $\boldsymbol{f} \in C_{c}^{\infty}(\Omega)^{m}$, let $\boldsymbol{u} \in Y^{1,2}(\Omega)^{m}$ be a unique weak solution of the problem

$$
\left\{\begin{array} { r l } 
{ L \boldsymbol { u } = \boldsymbol { f } } & { \text { in } \Omega , } \\
{ \boldsymbol { A } D \boldsymbol { u } \cdot \boldsymbol { n } = 0 } & { \text { on } \partial \Omega }
\end{array} \quad \text { or } \quad \left\{\begin{array}{rl}
{ }^{t} L \boldsymbol{u}=\boldsymbol{f} & \text { in } \Omega, \\
{ }^{t} \boldsymbol{A} D \boldsymbol{u} \cdot \boldsymbol{n}=0 & \text { on } \partial \Omega .
\end{array}\right.\right.
$$


Then for all $x \in \Omega$ and $R>0$, we have

$$
\|\boldsymbol{u}\|_{L^{\infty}\left(\Omega_{R / 2}(x)\right)} \leq C_{1}\left(R^{-d / 2}\|\boldsymbol{u}\|_{L^{2}\left(\Omega_{R}(x)\right)}+R^{2}\|\boldsymbol{f}\|_{L^{\infty}\left(\Omega_{R}(x)\right)}\right) .
$$

Theorem 5.2. Let $\Omega$ be a Lipschitz graph domain in $\mathbb{R}^{d}(d \geq 3)$ with Lipschitz constant $K$ and assume condition $\left(\underline{\mathrm{IH}^{\prime}}\right)$. If condition $\left(\underline{\mathrm{LB}^{\prime}}\right)$ is also satisfied, then conclusions of Theorem 3.6 hold with $C=C\left(d, m, \lambda, M, K, C_{1}\right)$. Conversely, suppose there exists a constant $C_{2}$ such that (3.10) holds. Then condition ([-1) is satisfied in $\Omega$ with $C_{1}=C_{1}\left(d, m, \lambda, M, K, C_{2}\right)$.

5.2. Proof of Theorem [5.1. The proof is a slight modification of that of Theorem [3.1. Let $y \in \Omega$ be fixed but arbitrary. For $\varepsilon>0$ and $k=1, \ldots, m$, let $\boldsymbol{v}=\boldsymbol{v}_{\varepsilon, y, k}$ be a unique weak solution in $Y^{1,2}(\Omega)^{m}$ of the problem

$$
\left\{\begin{aligned}
L \boldsymbol{v} & =\Phi_{\varepsilon} \boldsymbol{e}_{k} \text { in } \Omega \\
\boldsymbol{A D} \boldsymbol{v} \cdot \boldsymbol{n} & =0 \text { on } \partial \Omega
\end{aligned}\right.
$$

and define $\boldsymbol{N}^{\varepsilon}(\cdot, y)$ by (4.2). Then $\boldsymbol{N}^{\varepsilon}(\cdot, y)$ satisfies the identity

$$
\int_{\Omega} A_{i j}^{\alpha \beta} D_{\beta} N_{j k}^{\varepsilon}(\cdot, y) D_{\alpha} \phi^{i}=\int_{\Omega_{\varepsilon}(y)} \Phi_{\varepsilon} \phi^{k}, \quad \forall \phi \in Y^{1,2}(\Omega)^{m} .
$$

By the same argument as in the proof of Theorem 3.1, we then obtain (4.5). Let $R \in\left(0, d_{y}^{\prime}\right)$ be arbitrary, but fixed. Assume that $\boldsymbol{f} \in C_{c}^{\infty}(\Omega)^{m}$ is supported in $B_{R}=B_{R}(y) \subset \Omega$ and let $\boldsymbol{u}$ be a unique weak solution in $Y^{1,2}(\Omega)^{m}$ of the problem (2.16). Then, we get the identity (4.7) and also the estimate (4.8). By literally the same steps as in the proof of Theorem 3.1, we get (4.9) and (4.12) - 4.20) with $d_{y}$ replaced by $d_{y}^{\prime}$. Therefore, by the same reasoning as in the proof of Theorem 3.1, we find that $\boldsymbol{N}(\cdot, y)$ satisfies properties i) and ii) in Section 2.4 .2 and also the estimates i) - vii) in Theorem 3.1 with $d_{y}$ replaced by $d_{y}^{\prime}$; see [12, Section 4.1]. Let $\boldsymbol{u}$ be a unique solution in $Y^{1,2}(\Omega)^{m}$ of the problem (2.16) with $f \in C_{c}^{\infty}(\Omega)^{m}$. Then as in the proof of Theorem 3.1 again, we get (2.13). Therefore, $\boldsymbol{N}(x, y)$ satisfies property iii) in Section 2.4.2, and thus that $\boldsymbol{N}(x, y)$ is a unique Neumann function of the operator $L$ in $\Omega$. The proof is complete.

5.3. Proof of Theorem [5.2. We follow the proofs of Theorems 3.6 and 3.9 with a few adjustments. Let $y \in \Omega$ and $R>0$ be arbitrary, but fixed. Assume that $\boldsymbol{f} \in C_{c}^{\infty}(\Omega)^{m}$ is supported in $\Omega_{R}(y)$ and let $\boldsymbol{u}$ be a unique weak solution in $Y^{1,2}(\Omega)^{m}$ of the problem

$$
\left\{\begin{aligned}
{ }^{t} L \boldsymbol{u} & =\boldsymbol{f} \text { in } \Omega, \\
{ }^{t} \boldsymbol{A} D \boldsymbol{u} \cdot \boldsymbol{n} & =0 \text { on } \partial \Omega .
\end{aligned}\right.
$$

Then we have (4.7) and (4.8) as in the proof of Theorem 3.6 and thus by (2.2) we get (4.21). By ( $\left(\mathrm{LB}^{\prime}\right)$, 4.21), and Hölder's inequality, we obtain (4.23). Then by following the same steps as in the proof of Theorem 3.6 we get (4.25) with $C=C\left(d, m, \lambda, M, K, C_{1}\right)$.

Let $x \in \Omega, r>0$, and $\varepsilon>0$ be such that $B_{\varepsilon}(y) \cap B_{r}(x)=\emptyset$. Since the $k$-th column of $\boldsymbol{N}^{\varepsilon}(\cdot, y)$ is a unique weak solution in $Y^{1,2}(\Omega)^{m}$ of problem (5.3), condition $\left(\underline{\mathrm{LB}^{\prime}}\right)$ implies that

$$
\left\|\boldsymbol{N}^{\varepsilon}(\cdot, y)\right\|_{L^{\infty}\left(\Omega_{r / 2}(x)\right)} \leq C_{1} r^{-d / 2}\left\|\boldsymbol{N}^{\varepsilon}(\cdot, y)\right\|_{L^{2}\left(\Omega_{r}(x)\right)} .
$$

Then by following literally the same steps as in the proof of Theorem 3.6 , we obtain the desired pointwise bound (3.7). 
Next, let $\boldsymbol{v}_{\varepsilon}$ be the $k$-th column of $\boldsymbol{N}^{\varepsilon}(\cdot, y)$, where $k=1, \ldots, m, 0<\varepsilon<$ $\min \left(d_{y}^{\prime}, r\right) / 6$, and $r>0$. Let $\eta$ be a smooth function on $\mathbb{R}^{d}$ satisfying the conditions in (4.10). We set $\phi=\eta^{2} \boldsymbol{v}_{\varepsilon}$ in (5.4) to get Caccioppoli's inequality

$$
\int_{\Omega} \eta^{2}\left|D \boldsymbol{N}^{\varepsilon}(\cdot, y)\right|^{2} \leq C \int_{\Omega}|D \eta|^{2}\left|\boldsymbol{N}^{\varepsilon}(\cdot, y)\right|^{2} .
$$

By using the conditions in (4.10) for $\eta$ and the pointwise bound (3.7), we get

$$
\int_{\Omega \backslash B_{r}(y)}\left|D \boldsymbol{N}^{\varepsilon}(\cdot, y)\right|^{2} \leq C r^{-2} \int_{B_{r}(y) \backslash B_{r / 2}(y)}|x-y|^{4-2 d} d x \leq C r^{2-d} .
$$

Therefore, by taking the limit $\varepsilon \rightarrow 0$, we get

$$
\|D N(\cdot, y)\|_{L^{2}\left(\Omega \backslash B_{r}(y)\right)} \leq C r^{(2-d) / 2}, \quad \forall r>0 .
$$

The pointwise bound (3.7) together with the above estimate yields

$$
\|\boldsymbol{N}(\cdot, y)\|_{L^{2 d /(d-2)}\left(\Omega \backslash B_{r}(y)\right)}+\|D \boldsymbol{N}(\cdot, y)\|_{L^{2}\left(\Omega \backslash B_{r}(y)\right)} \leq C r^{(2-d) / 2}, \quad \forall r>0,
$$

where $C=C\left(d, m, \lambda, M, K, C_{1}\right)$. By following literally the same step used in deriving (4.15) - (4.18) from (4.14) we obtain from (5.6) the estimates i) - v) in Theorem 3.6 with constants $C=C\left(d, m, \lambda, M, K, C_{1}\right)$.

It remains to show that the pointwise bound (3.7) implies condition ( $\left(\mathrm{LB}^{\prime}\right)$. By symmetry, it is enough to prove $\left(\underline{\mathrm{LB}^{\prime}}\right)$ for a weak solution $\boldsymbol{u} \in Y^{1,2}(\Omega)^{m}$ of the problem

$$
\left\{\begin{aligned}
{ }^{t} L \boldsymbol{u}=\boldsymbol{f} & \text { in } \Omega, \\
{ }^{t} \boldsymbol{A} D \boldsymbol{u} \cdot \boldsymbol{n}=0 & \text { on } \partial \Omega .
\end{aligned}\right.
$$

Let $\boldsymbol{u}$ be a unique weak solution in $Y^{1,2}(\Omega)^{m}$ of the above problem, where $\boldsymbol{f} \in$ $C_{c}^{\infty}(\Omega)^{m}$, so that we have the identity

$$
\int_{\Omega} A_{i j}^{\alpha \beta} D_{\beta} w^{j} D_{\alpha} u^{i}=\int_{\Omega} f^{i} w^{i}, \quad \forall \boldsymbol{w} \in Y^{1,2}(\Omega)^{m} .
$$

We set $\boldsymbol{w}=\zeta \boldsymbol{v}_{\varepsilon}$ in (5.7), where $\zeta$ is as in (4.32) and $\boldsymbol{v}_{\varepsilon}$ is the weak solution in $Y^{1,2}(\Omega)^{m}$ of problem (5.3) (i.e., $\boldsymbol{v}_{\varepsilon}$ is the $k$-th column of $\boldsymbol{N}^{\varepsilon}(\cdot, y)$ ), to get

$$
\int_{\Omega} \zeta A_{i j}^{\alpha \beta} D_{\beta} v_{\varepsilon}^{j} D_{\alpha} u^{i}=-\int_{\Omega} A_{i j}^{\alpha \beta} D_{\beta} \zeta v_{\varepsilon}^{j} D_{\alpha} u^{i}+\int_{\Omega} \zeta f^{i} v_{\varepsilon}^{i} .
$$

On the other hand, by setting $\phi=\zeta \boldsymbol{u}$ in (5.4), we get

$$
\int_{\Omega_{\varepsilon}(y)} \Phi_{\varepsilon} \zeta u^{k}=\int_{\Omega} \zeta A_{i j}^{\alpha \beta} D_{\beta} v_{\varepsilon}^{j} D_{\alpha} u^{i}+\int_{\Omega} A_{i j}^{\alpha \beta} D_{\beta} v_{\varepsilon}^{j} D_{\alpha} \zeta u^{i} .
$$

Therefore, by combining (5.8) and (5.9), we obtain

$$
\int_{\Omega_{\varepsilon}(y)} \Phi_{\varepsilon} \zeta u^{k}=\int_{\Omega} A_{i j}^{\alpha \beta} D_{\beta} v_{\varepsilon}^{j} D_{\alpha} \zeta u^{i}-\int_{\Omega} A_{i j}^{\alpha \beta} D_{\beta} \zeta v_{\varepsilon}^{j} D_{\alpha} u^{i}+\int_{\Omega} \zeta f^{i} v_{\varepsilon}^{i} .
$$

Assume $y \in \Omega_{R / 4}(x)$ and take $\varepsilon \rightarrow 0$ in the above identity to get (cf. (4.35) )

$$
\begin{aligned}
u^{k}(y)=\int_{\Omega} A_{i j}^{\alpha \beta} D_{\beta} N_{j k}(\cdot, y) D_{\alpha} \zeta u^{i}-\int_{\Omega} A_{i j}^{\alpha \beta} & D_{\beta} \zeta N_{j k}(\cdot, y) D_{\alpha} u^{i} \\
& +\int_{\Omega} \zeta f^{i} N_{j k}(\cdot, y)=: I_{1}+I_{2}+I_{3} .
\end{aligned}
$$

On the other hand, by using the fact that $C_{c}^{\infty}(\bar{\Omega})$ is dense in $Y^{1,2}(\Omega)$ (see the proof of Lemma 6.8 in the Appendix), we may set $\phi=\eta^{2} \boldsymbol{v}$ in (2.15), where $\boldsymbol{v}$ is the 
$k$-th column of $\boldsymbol{N}(\cdot, y)$ and $\eta$ satisfies the properties in (4.10), to get the following inequality (cf. (5.5) ):

$$
\int_{\Omega} \eta^{2}|D \mathbf{N}(\cdot, y)|^{2} \leq C \int_{\Omega}|D \eta|^{2}|\mathbf{N}(\cdot, y)|^{2} .
$$

Then by proceeding as before, we again obtain the estimate (5.6). With the aid of (2.2), we also derive the following Caccioppoli inequality from (5.7):

$$
\|D \boldsymbol{u}\|_{L^{2}\left(\Omega_{R / 2}(x)\right)} \leq C R^{-1}\|\boldsymbol{u}\|_{L^{2}\left(\Omega_{R}(x)\right)}+C\|\boldsymbol{f}\|_{L^{2 d /(d+2)}\left(\Omega_{R}(x)\right)} .
$$

Now, denote $A_{R}(y)=\Omega_{3 R / 4}(y) \backslash B_{R / 8}(y)$. Recall that $\zeta$ satisfies the properties in (4.32). Then by Hölder's inequality and (5.6), we estimate

$$
\left|I_{1}\right| \leq C R^{-1}\|D N(\cdot, y)\|_{L^{2}\left(A_{R}(y)\right)}\|\boldsymbol{u}\|_{L^{2}\left(\Omega_{R / 2}(x)\right)} \leq C R^{-d / 2}\|\boldsymbol{u}\|_{L^{2}\left(\Omega_{R}(x)\right)} .
$$

Similarly, by Hölder's inequality, (5.6), and (5.10), we estimate

$$
\begin{aligned}
\left|I_{2}\right| & \leq C R^{-1}\|\boldsymbol{N}(\cdot, y)\|_{L^{2}\left(A_{R}(y)\right)}\|D \boldsymbol{u}\|_{L^{2}\left(\Omega_{R / 2}(x)\right)} \\
& \leq C R^{-d / 2}\|\boldsymbol{u}\|_{L^{2}\left(\Omega_{R}(x)\right)}+R^{2}\|\boldsymbol{f}\|_{L^{\infty}\left(\Omega_{R}(x)\right)} .
\end{aligned}
$$

Finally, by Hölder's inequality and (3.10), we estimate

$$
\left|I_{3}\right| \leq C\|\boldsymbol{N}(\cdot, y)\|_{L^{1}\left(\Omega_{3 R / 4}(y)\right)}\|\boldsymbol{f}\|_{L^{\infty}\left(\Omega_{R / 2}(x)\right)} \leq C R^{2}\|\boldsymbol{f}\|_{L^{\infty}\left(\Omega_{R}(x)\right)} .
$$

Combining the above estimates and using a standard covering argument, we obtain $\left(\underline{\mathrm{LB}^{\prime}}\right)$. The proof is complete.

\section{Appendix}

Lemma 6.1. Let $\Omega$ be a bounded $C^{1}$ domain in $\mathbb{R}^{d}$. Suppose that the coefficients $A_{i j}^{\alpha \beta}$ of system (1.1) belong to the VMO class and satisfy conditions (2.3) and (2.4). Then condition (LB) is satisfied.

Proof. Assume that $\boldsymbol{f} \in C_{c}^{\infty}(\Omega)^{m}$ and $\boldsymbol{g} \in C^{\infty}(\partial \Omega)^{m}$ satisfy the compatibility condition $\int_{\Omega} \boldsymbol{f}+\int_{\partial \Omega} \boldsymbol{g}=0$ and let $\boldsymbol{u} \in \tilde{W}^{1,2}(\Omega)^{m}$ be a unique weak solution of the problem

$$
\left\{\begin{aligned}
L \boldsymbol{u} & =\boldsymbol{f} \text { in } \Omega, \\
\boldsymbol{A} D \boldsymbol{u} \cdot \boldsymbol{n} & =\boldsymbol{g} \text { on } \partial \Omega .
\end{aligned}\right.
$$

Let $\boldsymbol{v}=\zeta \boldsymbol{u}$, where $\zeta: \mathbb{R}^{d} \rightarrow \mathbb{R}$ is a smooth function to be chosen later, and observe that $\boldsymbol{v}$ is a weak solution in $W^{1,2}(\Omega)^{m}$ of the problem

$$
\left\{\begin{aligned}
-D_{\alpha}\left(A_{i j}^{\alpha \beta} D_{\beta} v^{j}\right) & =\zeta f^{i}-\Psi^{i}-D_{\alpha} F_{\alpha}^{i} \text { in } \Omega, \\
A_{i j}^{\alpha \beta} D_{\beta} v^{j} n_{\alpha} & =\zeta g^{i}+F_{\alpha}^{i} n_{\alpha} \text { on } \partial \Omega,
\end{aligned}\right.
$$

where we used the notation

$$
\Psi^{i}=A_{i j}^{\alpha \beta} D_{\alpha} \eta D_{\beta} u^{j}, \quad F_{\alpha}^{i}=A_{i j}^{\alpha \beta} D_{\beta} \zeta u^{j} .
$$

For $i=1, \ldots, m$, let $w^{i}$ be a solution of the Neumann problem

$$
\left\{\begin{aligned}
-\Delta w^{i} & =\zeta f^{i}-\Psi^{i} \quad \text { in } \Omega, \\
\partial w^{i} / \partial n & =\zeta g^{i} \quad \text { on } \partial \Omega .
\end{aligned}\right.
$$


Then, by [9, Corollary 9.3] together with the embedding theorems of Sobolev and Besov spaces (see, e.g., 4]), we have the following estimate for $D w^{i}$ provided $p>$ $d /(d-1)$ :

$$
\|D \boldsymbol{w}\|_{L^{p}(\Omega)} \leq C\left(\|\zeta \boldsymbol{f}\|_{L^{p d /(p+d)}(\Omega)}+\|\boldsymbol{\Psi}\|_{L^{p d /(p+d)}(\Omega)}+\|\zeta \boldsymbol{g}\|_{L^{p(d-1) / d}(\partial \Omega)}\right) .
$$

Notice that if we set $h_{\alpha}^{i}=D_{\alpha} w^{i}+F_{\alpha}^{i}$, then $\boldsymbol{v}$ becomes a weak solution of the problem

$$
\left\{\begin{aligned}
D_{\alpha}\left(A_{i j}^{\alpha \beta} D_{\beta} v^{j}\right) & =D_{\alpha} h_{\alpha}^{i} \text { in } \Omega, \\
\left(A_{i j}^{\alpha \beta} D_{\beta} v^{j}-h_{\alpha}^{i}\right) n_{\alpha} & =0 \text { on } \partial \Omega .
\end{aligned}\right.
$$

We then apply [5, Theorem 1] to conclude that $\boldsymbol{v} \in W^{1, p}(\Omega)^{m}$ with the estimate (6.2)

$\|D \boldsymbol{v}\|_{L^{p}(\Omega)} \leq C\left(\|\zeta \boldsymbol{f}\|_{L^{p d /(p+d)}(\Omega)}+\|\boldsymbol{\Psi}\|_{L^{p d /(p+d)}(\Omega)}+\|\zeta \boldsymbol{g}\|_{L^{p(d-1) / d}(\partial \Omega)}+\|\boldsymbol{F}\|_{L^{p}(\Omega)}\right)$.

By choosing $\zeta \equiv 1$, we find that $\boldsymbol{u} \in W^{1, p}(\Omega)^{m}$ and

$$
\|D \boldsymbol{u}\|_{L^{p}(\Omega)} \leq C\left(\|\boldsymbol{f}\|_{L^{p d /(p+d)}(\Omega)}+\|\boldsymbol{g}\|_{L^{p(d-1) / d}(\partial \Omega)}\right),
$$

and thus, via Morrey's imbedding theorem, we find that $\boldsymbol{u} \in C^{\mu}(\bar{\Omega})$ for any $\mu \in$ $(0,1)$, which particularly implies that $\boldsymbol{u}$ is globally bounded in $\Omega$.

To obtain ( $(\overline{\mathrm{LB}})$, we employ the standard localization method as follows. Let $x \in \Omega$ and $0<R<\operatorname{diam}(\Omega)$ be arbitrary but fixed. For any $y \in \Omega \cap B_{R}(x)$ and $0<\rho<r \leq R$, we choose the function $\zeta$ such that

$$
0 \leq \zeta \leq 1, \quad \operatorname{supp} \zeta \subset B_{r}(y), \quad \zeta \equiv 1 \text { on } B_{\rho}(y), \quad \text { and } \quad|D \zeta| \leq 2 /(r-\rho) .
$$

Recall that we use the notation

$$
\Omega_{r}=\Omega_{r}(y)=\Omega \cap B_{r}(y), \quad \Sigma_{r}=\Sigma_{r}(y)=\partial \Omega \cap B_{r}(y) .
$$

Then by using the assumptions on $\zeta$, we estimate terms in (6.2) as follows:

$$
\begin{aligned}
\|\zeta \boldsymbol{f}\|_{L^{p d /(p+d)}(\Omega)} & \leq C r^{1+d / p}\|\boldsymbol{f}\|_{L^{\infty}\left(\Omega_{r}\right)}, \\
\|\boldsymbol{\Psi}\|_{L^{p d /(p+d)}(\Omega)} & \leq C(r-\rho)^{-1}\|D \boldsymbol{u}\|_{L^{p d /(p+d)}\left(\Omega_{r}\right)}, \\
\|\zeta \boldsymbol{g}\|_{L^{p(d-1) / d}(\Omega)} & \leq C r^{d / p}\|\boldsymbol{g}\|_{L^{\infty}\left(\Sigma_{r}\right)} \\
\|\boldsymbol{F}\|_{L^{p}(\Omega)} & \leq C(r-\rho)^{-1}\|\boldsymbol{u}\|_{L^{p}\left(\Omega_{r}\right)}
\end{aligned}
$$

By using inequality (6.2) and the above estimates, we get

$$
\begin{aligned}
\|D \boldsymbol{u}\|_{L^{p}\left(\Omega_{\rho}\right)} \leq C r^{1+d / p}\|\boldsymbol{f}\|_{L^{\infty}\left(\Omega_{r}\right)}+C r^{d / p}\|\boldsymbol{g}\|_{L^{\infty}\left(\Sigma_{r}\right)} & \\
& +C(r-\rho)^{-1}\|\boldsymbol{u}\|_{L^{p}\left(\Omega_{r}\right)}+C(r-\rho)^{-1}\|D \boldsymbol{u}\|_{L^{p d /(p+d)}\left(\Omega_{r}\right)} .
\end{aligned}
$$

We fix $p>d$ and let $k$ be the smallest integer such that $k \geq d(1 / 2-1 / p)$. We set

$$
p_{i}=p d /(d+p i) \quad \text { and } \quad r_{i}=\rho+(r-\rho) i / k, \quad i=0, \ldots, k .
$$

Then we apply (6.3) iteratively to get

$$
\begin{aligned}
\|D \boldsymbol{u}\|_{L^{p}\left(\Omega_{\rho}\right)} \leq & \sum_{i=1}^{k} C^{i}\left(\frac{k}{r-\rho}\right)^{i-1}\left(r_{i}^{1+d / p_{i-1}}\|\boldsymbol{f}\|_{L^{\infty}\left(\Omega_{r_{i}}\right)}+r_{i}^{d / p_{i-1}}\|\boldsymbol{g}\|_{L^{\infty}\left(\Sigma_{r_{i}}\right)}\right) \\
& +\sum_{i=1}^{k} C^{i}\left(\frac{k}{r-\rho}\right)^{i}\|\boldsymbol{u}\|_{L^{p_{i-1}\left(\Omega_{r_{i}}\right.}}+C^{k}\left(\frac{k}{r-\rho}\right)^{k}\|D \boldsymbol{u}\|_{L^{p_{k}\left(\Omega_{r_{k}}\right.}} .
\end{aligned}
$$


Notice that $1<p_{k} \leq 2$. By using Hölder's inequality we then obtain

$$
\begin{array}{r}
\rho^{-d(1 / 2-1 / p)}\|D \boldsymbol{u}\|_{L^{2}\left(\Omega_{\rho}\right)} \leq C\left(\frac{r}{r-\rho}\right)^{k-1}\left(r^{1+d / p}\|\boldsymbol{f}\|_{L^{\infty}\left(\Omega_{r}\right)}+r^{d / p}\|\boldsymbol{g}\|_{L^{\infty}\left(\Sigma_{r}\right)}\right) \\
+C\left(\frac{r}{r-\rho}\right)^{k} r^{-1}\|\boldsymbol{u}\|_{L^{p}\left(\Omega_{r}\right)}+C\left(\frac{r}{r-\rho}\right)^{k} r^{d(1 / p-1 / 2)}\|D \boldsymbol{u}\|_{L^{2}\left(\Omega_{r}\right)} .
\end{array}
$$

If we take $r=R / 4$ and $\rho<r / 2=R / 4$ in the above, then for all $y \in \Omega_{R / 4}(x)$, we get

$$
\begin{gathered}
\left(\rho^{-(d-2+2(1-d / p))} \int_{\Omega_{\rho}(y)}|D \boldsymbol{u}|^{2}\right)^{1 / 2} \leq C R^{1+d / p}\|\boldsymbol{f}\|_{L^{\infty}\left(\Omega_{R}(x)\right)}+C R^{d / p}\|\boldsymbol{g}\|_{L^{\infty}\left(\Sigma_{R}(x)\right)} \\
+C R^{-1}\|\boldsymbol{u}\|_{L^{p}\left(\Omega_{R}(x)\right)}+C R^{d(1 / p-1 / 2)}\|D \boldsymbol{u}\|_{L^{2}\left(\Omega_{R / 2}(x)\right)}=: A(R) .
\end{gathered}
$$

Hereafter in the proof we shall denote $\Omega_{R}=\Omega_{R}(x)$. Then by Morrey-Campanato's theorem (see [10, Section 3.1]), for all $z, z^{\prime} \in \Omega_{R / 4}$, we have

$$
\left|\boldsymbol{u}(z)-\boldsymbol{u}\left(z^{\prime}\right)\right| \leq C R^{1-d / p} A(R),
$$

where $A(R)$ is as defined in (6.4). Therefore, for any $z \in \Omega_{R / 4}$ we have

$$
|\boldsymbol{u}(z)| \leq\left|\boldsymbol{u}\left(z^{\prime}\right)\right|+\left|\boldsymbol{u}(z)-\boldsymbol{u}\left(z^{\prime}\right)\right| \leq\left|\boldsymbol{u}\left(z^{\prime}\right)\right|+C R^{1-d / p} A(R), \quad \forall z^{\prime} \in \Omega_{R / 4} .
$$

By taking average over $z^{\prime} \in \Omega_{R / 4}$ in the above and using the definition of $A(R)$, we obtain

$$
\begin{aligned}
\sup _{\Omega_{R / 4}}|\boldsymbol{u}| \leq f_{\Omega_{R / 4}}\left|\boldsymbol{u}\left(z^{\prime}\right)\right| d z^{\prime}+C & R^{2}\|\boldsymbol{f}\|_{L^{\infty}\left(\Omega_{R}\right)}+C R\|\boldsymbol{g}\|_{L^{\infty}\left(\Sigma_{R}\right)} \\
& +C R^{-d / p}\|\boldsymbol{u}\|_{L^{p}\left(\Omega_{R}\right)}+C R^{1-d / 2}\|D \boldsymbol{u}\|_{L^{2}\left(\Omega_{R / 2}\right)} .
\end{aligned}
$$

Then by using Hölder's inequality, Caccioppoli's inequality (see Lemma 6.5 below), and the fact that $\Omega$ is bounded, we get

$\sup _{\Omega_{R / 4}}|\boldsymbol{u}| \leq C R^{2}\|\boldsymbol{f}\|_{L^{\infty}\left(\Omega_{R}\right)}+C R\|\boldsymbol{g}\|_{L^{\infty}\left(\Sigma_{R}\right)}+C R^{-d / p}\|\boldsymbol{u}\|_{L^{p}\left(\Omega_{R}\right)}+C R^{-d / 2}\|\boldsymbol{u}\|_{L^{2}\left(\Omega_{R}\right)}$.

By using a standard argument (see [10, pp. 80-82]), we derive from the above inequality

$$
\sup _{\Omega_{R / 2}}|\boldsymbol{u}| \leq C R^{2}\|\boldsymbol{f}\|_{L^{\infty}\left(\Omega_{R}\right)}+C R\|\boldsymbol{g}\|_{L^{\infty}\left(\Sigma_{R}\right)}+C R^{-d / 2}\|\boldsymbol{u}\|_{L^{2}\left(\Omega_{R}\right)} .
$$

The proof is complete.

Lemma 6.5. Let $\Omega \subset \mathbb{R}^{d}$ be a bounded Lipschitz domain. Let $\boldsymbol{u} \in W^{1,2}(\Omega)^{m}$ be a weak solution of the problem

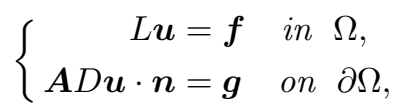

where $\boldsymbol{f} \in L^{\infty}(\Omega)^{m}$ and $\boldsymbol{g} \in L^{\infty}(\partial \Omega)^{m}$. Then we have

$\|D \boldsymbol{u}\|_{L^{2}\left(\Omega_{R / 2}\right)} \leq C R^{-1}\|\boldsymbol{u}\|_{L^{2}\left(\Omega_{R}\right)}+C R^{d / 2}(1+R)\|\boldsymbol{g}\|_{L^{\infty}\left(\Sigma_{R}\right)}+C R^{d / 2+1}\|\boldsymbol{f}\|_{L^{\infty}\left(\Omega_{R}\right)}$, where $C=C(d, m, \lambda, M, \Omega)$. 
Proof. Let $\eta$ be a smooth function on $\mathbb{R}^{d}$ satisfying

$$
0 \leq \eta \leq 1, \quad \operatorname{supp} \eta \subset B_{R}, \quad \eta \equiv 1 \text { on } B_{R / 2}, \quad \text { and } \quad|D \eta| \leq 4 / R .
$$

By setting $\boldsymbol{v}=\eta^{2} \boldsymbol{u}$ in (2.10), we obtain

$$
\int_{\Omega} \eta^{2} A_{i j}^{\alpha \beta} D_{\beta} u^{j} D_{\alpha} u^{i}=-\int_{\Omega} 2 \eta A_{i j}^{\alpha \beta} D_{\beta} \eta u^{j} D_{\alpha} u^{i}+\int_{\partial \Omega} \eta^{2} g^{i} u^{i}+\int_{\Omega} \eta^{2} f^{i} u^{i} .
$$

Then by (2.3), (2.4), and Cauchy's inequality, we get

$$
\int_{\Omega} \eta^{2}|D \boldsymbol{u}|^{2} \leq C \int_{\Omega}|D \eta|^{2}|\boldsymbol{u}|^{2}+C\|\boldsymbol{g}\|_{L^{\infty}\left(\Sigma_{R}\right)}\|\eta \boldsymbol{u}\|_{L^{1}(\partial \Omega)}+C\|\boldsymbol{f}\|_{L^{\infty}\left(\Omega_{R}\right)}\|\boldsymbol{u}\|_{L^{1}\left(\Omega_{R}\right)} .
$$

Observe that the trace theorem (see, e.g., 8] ) yields

$$
\int_{\partial \Omega}|\eta \boldsymbol{u}| \leq C \int_{\Omega}|D(\eta \boldsymbol{u})|+|\eta \boldsymbol{u}| \leq C \int_{\Omega}|D \eta||\boldsymbol{u}|+\eta|D \boldsymbol{u}|+|\eta \boldsymbol{u}| .
$$

Therefore, by using Hölder's inequality and Cauchy's inequality, we estimate

$$
\begin{aligned}
& \|\boldsymbol{g}\|_{L^{\infty}\left(\Sigma_{R}\right)}\|\eta \boldsymbol{u}\|_{L^{1}(\partial \Omega)} \\
& \quad \leq C\|\boldsymbol{g}\|_{L^{\infty}\left(\Sigma_{R}\right)}\left(R^{d / 2-1}\|\boldsymbol{u}\|_{L^{2}\left(\Omega_{R}\right)}+C R^{d / 2}\|\eta D \boldsymbol{u}\|_{L^{2}\left(\Omega_{R}\right)}+C R^{d / 2}\|\boldsymbol{u}\|_{L^{2}\left(\Omega_{R}\right)}\right) \\
& \quad \leq C R^{d}\left(1+\varepsilon^{-1}+R^{2}\right)\|\boldsymbol{g}\|_{L^{\infty}\left(\Sigma_{R}\right)}^{2}+C R^{-2}\|\boldsymbol{u}\|_{L^{2}\left(\Omega_{R}\right)}^{2}+\varepsilon \int_{\Omega} \eta^{2}|D \boldsymbol{u}|^{2} .
\end{aligned}
$$

Similarly, by Hölder's inequality and Cauchy's inequality, we obtain

$$
\begin{aligned}
\|\boldsymbol{f}\|_{L^{\infty}\left(\Omega_{R}\right)}\|\boldsymbol{u}\|_{L^{1}\left(\Omega_{R}\right)} & \leq C R^{d / 2}\|\boldsymbol{f}\|_{L^{\infty}\left(\Omega_{R}\right)}\|\boldsymbol{u}\|_{L^{2}\left(\Omega_{R}\right)} \\
& \leq C R^{d+2}\|\boldsymbol{f}\|_{L^{\infty}\left(\Omega_{R}\right)}^{2}+C R^{-2}\|\boldsymbol{u}\|_{L^{2}\left(\Omega_{R}\right)}^{2} .
\end{aligned}
$$

By combining (6.7) and the above inequality, we get

$$
\|D \boldsymbol{u}\|_{L^{2}\left(\Omega_{R / 2}\right)}^{2} \leq C R^{-2}\|\boldsymbol{u}\|_{L^{2}\left(\Omega_{R}\right)}^{2}+C R^{d}\left(1+R^{2}\right)\|\boldsymbol{g}\|_{L^{\infty}\left(\Sigma_{R}\right)}^{2}+C R^{d+2}\|\boldsymbol{f}\|_{L^{\infty}\left(\Omega_{R}\right)}^{2} .
$$

The above inequality obviously yields (6.6).

Lemma 6.8. Let $\Omega \subset \mathbb{R}^{d}$ be a Lipschitz graph domain with Lipschitz constant $K$. Then, for any $u \in Y^{1,2}(\Omega)$, we have

$$
\|u\|_{L^{2 d /(d-2)}(\Omega)} \leq C(d, K)\|D u\|_{L^{2}(\Omega)} .
$$

Proof. We begin by showing that $C_{c}^{\infty}(\bar{\Omega})$ is dense in $Y^{1,2}(\Omega)$. By following the same steps as in the proof of the approximation theorem for Sobolev functions (see, e.g., [8]), we find that $C^{\infty}(\bar{\Omega}) \cap Y^{1,2}(\Omega)$ is dense in $Y^{1,2}(\Omega)$. On the other hand, one can approximate $u \in C^{\infty}(\bar{\Omega}) \cap Y^{1,2}(\Omega)$ by a sequence of functions from $C_{c}^{\infty}(\bar{\Omega})$ in the $Y^{1,2}(\Omega)$ norm as follows. For each $k=1,2, \ldots$, let $\phi_{k} \in C_{c}^{\infty}\left(\mathbb{R}^{d}\right)$ be such that

$$
0 \leq \phi_{k} \leq 1, \quad \phi_{k}=1 \quad \text { on } B_{k}(0), \quad \operatorname{supp} \phi_{k} \subset B_{3 k}(0), \quad \text { and }\left|\nabla \phi_{k}\right| \leq 1 / k .
$$

Then, obviously $u \phi_{k} \in C_{c}^{\infty}(\bar{\Omega})$, and it is easy to check $\left\|u \phi_{k}-u\right\|_{Y^{1,2}(\Omega)} \rightarrow 0$ as $k \rightarrow \infty$. We have thus shown that $C_{c}^{\infty}(\bar{\Omega})$ is dense in $Y^{1,2}(\Omega)$. By essentially the same argument, we also find that $C_{c}^{\infty}\left(\mathbb{R}^{d}\right)$ is dense in $Y^{1,2}\left(\mathbb{R}^{d}\right)$. Therefore, the Sobolev inequality yields that

$$
\|u\|_{L^{2 d /(d-2)}\left(\mathbb{R}^{d}\right)} \leq C(d)\|D u\|_{L^{2}\left(\mathbb{R}^{d}\right)}, \quad \forall u \in Y^{1,2}\left(\mathbb{R}^{d}\right) .
$$


Next, we claim that there exists a bounded linear operator $E: Y^{1,2}(\Omega) \rightarrow Y^{1,2}\left(\mathbb{R}^{d}\right)$ such that $E u=u$ in $\Omega$ and

$$
\|D(E u)\|_{L^{2}\left(\mathbb{R}^{d}\right)} \leq C(d, K)\|D u\|_{L^{2}(\Omega)} .
$$

To prove (6.11), we follow the same steps in the usual proof of the extension theorem for Sobolev functions in Lipschitz domain (see, e.g., [8]). For $u \in C_{c}^{\infty}(\bar{\Omega})$, set

$$
\begin{aligned}
& u^{+}(y)=u(y) \text { if } y \in \bar{\Omega}, \\
& u^{-}(y)=u\left(y^{\prime}, 2 \gamma\left(y^{\prime}\right)-y_{d}\right) \text { if } y \in \mathbb{R}^{d} \backslash \Omega .
\end{aligned}
$$

Note $u^{-}=u^{+}=u$ on $\partial \Omega$. Then, it is routine to check (see [8, Section 4.1])

$$
\left\|D u^{-}\right\|_{L^{2}\left(\mathbb{R}^{d} \backslash \bar{\Omega}\right)} \leq C(K)\|D u\|_{L^{2}(\Omega)},
$$

and thus, we have

$$
\left\|u^{-}\right\|_{Y^{1,2}\left(\mathbb{R}^{d} \backslash \bar{\Omega}\right)} \leq C(K)\|u\|_{Y^{1,2}(\Omega)} .
$$

Define

$$
E u \equiv \bar{u} \equiv \begin{cases}u^{+} & \text {on } \bar{\Omega}, \\ u^{-} & \text {on } \mathbb{R}^{d} \backslash \Omega,\end{cases}
$$

and note that $\bar{u}$ is continuous on $\mathbb{R}^{d}$. Also, it is easy to see $\bar{u} \in Y^{1,2}\left(\mathbb{R}^{d}\right)$ and

$$
D \bar{u}= \begin{cases}D u^{+} & \text {on } \bar{\Omega}, \\ D u^{-} & \text {on } \mathbb{R}^{d} \backslash \Omega .\end{cases}
$$

Therefore, we have proved (6.11) in the case when $u \in C_{c}^{\infty}(\bar{\Omega})$. Since $C_{c}^{\infty}(\bar{\Omega})$ is dense in $Y^{1,2}(\Omega)$, we obtain (6.11) by the standard approximation argument. Finally, we obtain (6.9) by combining (6.10) and (6.11).

\section{ACKNOWLEDGMENT}

The authors thank Russell Brown and Hongjie Dong for their valuable discussions and comments. The authors would also like to thank the referee for helpful suggestions. This work was supported by the Basic Science Research Program through the National Research Foundation of Korea (NRF) funded by the Ministry of Education, Science and Technology (2010-0008224). The second author was supported by the WCU (World Class University) program through the National Research Foundation of Korea (NRF) funded by the Ministry of Education, Science and Technology (R31-10049) and also by a TJ Park Junior Faculty Fellowship.

\section{REFERENCES}

[1] Alfonseca, M. A.; Auscher, P.; Axelsson, A.; Hofmann, S.; Kim, S. Analyticity of layer potentials and $L^{2}$ solvability of boundary value problems for divergence form elliptic equations with complex $L^{\infty}$ coefficients. Adv. Math. 226 (2011), no. 5, 4533-4606. MR 2770458 (2012g:35055)

[2] Auscher, P.; Axelsson, A. Weighted maximal regularity estimates and solvability of nonsmooth elliptic systems I. Invent. Math. 184 (2011), no. 1, 47-115. MR2782252(2012c:35111)

[3] Auscher, P.; Axelsson, A.; Hofmann, S. Functional calculus of Dirac operators and complex perturbations of Neumann and Dirichlet problems. J. Funct. Anal. 255 (2008), no. 2, 374-448. MR2419965 (2009h:35079)

[4] Bergh, J.; Löfström, J. Interpolation spaces: An introduction. Springer-Verlag, Berlin-New York, 1976. MR0482275 (58:2349)

[5] Byun, S.; Chen, H.; Kim, M.; Wang, L. L $L^{p}$ regularity theory for linear elliptic systems. Discrete Contin. Dyn. Syst. 18 (2007), no. 1, 121-134. MR2276490 (2007m:35045) 
[6] Cho, S.; Dong, H.; Kim, S. Global estimates for Green's matrix of second order parabolic systems with application to elliptic systems in two dimensional domains. Potential Anal. 36 (2012), no. 2, 339-372. MR2886465

[7] Dong, H.; Kim, S. Green's matrices of second order elliptic systems with measurable coefficients in two dimensional domains. Trans. Amer. Math. Soc. 361 (2009), no. 6, 3303-3323. MR2485428(2010h:35005)

[8] Evans, L. C.; Gariepy, R. F. Measure theory and fine properties of functions. CRC Press, Boca Raton, FL, 1992. MR.1158660 (93f:28001)

[9] Fabes, E.; Mendez, O.; Mitrea, M. Boundary Layers on Sobolev-Besov Spaces and Poisson's Equation for the Laplacian in Lipschitz Domain. J. Funct. Anal. 159 (1998), no. 2, 323-368. MR:1658089 (99j:35036)

[10] Giaquinta, M. Introduction to regularity theory for nonlinear elliptic systems. Birkhäuser Verlag, Basel, 1993. MR1239172 (94g:49002)

[11] Grüter, M.; Widman, K.-O. The Green function for uniformly elliptic equations. Manuscripta Math. 37 (1982), no. 3, 303-342. MR657523 (83h:35033)

[12] Hofmann, S.; Kim, S. The Green function estimates for strongly elliptic systems of second order. Manuscripta Math. 124 (2007), no. 2, 139-172. MR2341783 (2008k:35110)

[13] Kang, K.; Kim, S. Global pointwise estimates for Green's matrix of second order elliptic systems. J. Differential Equations 249 (2010), no. 11, 2643-2662. MR2718661 (2011h:35056)

[14] Kenig, C. E. Harmonic analysis techniques for second order elliptic boundary value problems. American Mathematical Society, Providence, RI, 1994. MR1282720 (96a:35040)

[15] Kenig, C. E.; Lin, F.; Shen, Z. Homogenization of Elliptic Systems With Neumann Boundary Conditions. arXiv:1010.6114v1 [math.AP]

[16] Kenig, C. E.; Pipher, J. The Neumann problem for elliptic equations with nonsmooth coeffcients. Invent. Math. 113 (1993), no. 3, 447-509. MR.1231834 (95b:35046)

[17] Littman, W.; Stampacchia, G.; Weinberger, H. F. Regular points for elliptic equations with discontinuous coefficients. Ann. Scuola Norm. Sup. Pisa (3) 17 (1963) 43-77. MR0161019 (28:4228)

[18] Miranda, C. Partial differential equations of elliptic type. Springer-Verlag, New York-Berlin, 1970. MR0284700(44:1924)

[19] Shen, Z. Weighted estimates in $L^{2}$ for Laplace's equation on Lipschitz domains. Trans. Amer. Math. Soc. 357 (2005), no. 7, 2843-2870. MR2139930 (2006b:35074)

[20] Taylor, J. L.; Kim, S.; Brown, R. M. The Green function for elliptic systems in two dimensions. arXiv:1205.1089v1 [math.AP]

Department of Mathematics, Yonsei University, Seoul 120-749, Republic of Korea E-mail address: cjg@yonsei.ac.kr

Department of Mathematics, Yonsei University, Seoul 120-749, Republic of Korea Current address: Department of Computational Science and Engineering, Yonsei University, Seoul 120-749, Republic of Korea

E-mail address: kimseick@yonsei.ac.kr 